\title{
Validación del nuevo cultivar del camote forrajero: Lactogénico
}

Validation of the new cultivar of lactogenic forage sweet potato

Armando Quispe Cáceres ${ }^{1}$

http://dx.doi.org/10.21503/CienciayDesarrollo.2006.v6.01

\section{RESUMEN}

Con la finalidad de evaluar la adaptación de 20 clones de camote (Pomoea batatas) (L.), de aptitud forrajera y doble propósito (forraje y raíces comestibles) a los agroecosistemas de bosques secos del valle Quiroz-Piura, se realizaron dos ensayos en parcela de agricultores con germoplasma mejorado proveniente del Centro Internacional de la Papa-CIP. En el experimento una primavera-verano (noviembre 2001-junio 2002), los rendimientos de follaje fluctúan de 26,66 a 16,24 t/ha; los clones DLP 2462, Tipo 3, Mohc, Helena y Toquecita destacaron con 26,66; 22,43; 22,39; 22,20 y 22,19 t/ha respectivamente, no existiendo diferencias estadísticas en la producción de follaje, clasificándose como forrajera, forrajera doble propósito, forrajera y raicera respectivamente. Los clones Toquecita, Tabón, Mohc, SR90.323 y camote Ruiz (testigo1) produjeron rendimientos de 98,79; 52,78; 38,57; 36,54 y 27,20 t/ha de raíces comestibles, con diferencias estadísticas altamente significativas. En el experimento dos invierno-primavera (junio-diciembre 2002) los rendimientos de follaje fluctúan de 58,33 a 883 t/ha; los clones Solapa (testigo 2), Kumala Blanco, Tipo 3, Dulce y DLP 2462, destacaron con rendimientos de 58,33; 55,$33 ; 46,33 ; 41,00$ y $38,08 \mathrm{t} / \mathrm{ha}$ respectivamente, con diferencias estadísticas altamente significativas; clasificándose todas como forrajeras. Los clones Solapa, Pikis, Tabón, SR-90.323 y Toquecita, tuvieron rendimientos de 32,58; 22,33; 20,75; 18,58 y 16,50 t/ha de raíces comestibles respectivamente, sin diferencia estadística. Los rendimientos de follaje y raíces comestibles obtenidos se pueden considerar como exitosos y permiten concluir preliminarmente, la diferente respuesta de producción y adaptación promisoria de los clones de camote forrajero y doble propósito a las condiciones del valle Quiroz - Piura, se sugiere continuar con los ensayos en otras épocas.

\section{ABSTRACT}

In order to evaluate adaptation of 20 clones of sweet potato (Pomoea batatas) (L)) of forage aptitude and double intention (eatable forage and roots)to the agroecosistemas of dry forests of Quiroz - Piura valley, 2 tests were made in agriculturists' parcel with improved germoplasma originating from the International Center of Potato CIP. In Experiment 1 Spring-Summer (November 2001- June 2002) The foliage yields fluctuate from 26,66 to 16,24 t/ha; The clones DLP 2462, Type 3, Mohc, Helena, and Toquecita emphasized respectively with 26,$66 ; 22,43 ; 22,39 ; 22,20$ and $22,19 \mathrm{t} / \mathrm{ha}$, not existing statistical differences in the foliage production, classifying themselves like forage, forage double intention, forage and raicera respectively. The

1. Ingeniero Agrónomo, Magister Secientiae / Director técnico del proyecto camote forrajero - Universidad Alas Peruanas 
clones Toquecita, Tabon, Mhc, SR-90.232 and sweet potato Ruiz (witness 1) produced yields of $98.79 ; 52,78 ; 38,57 ; 36.54$ and $27,20 \mathrm{t} /$ ha of eatable roots, with highly significant statistical differences. In Experiment 2 Winter-Spring (June - December 2002) The foliage yields fluctuate from 58,33 to $883 \mathrm{t} / \mathrm{ha}$, the Solapa clones (witness 2) white Kumala, Type 3 , Sweet and DLP 2464, emphasized with yields of 58,$33 ; 55,33,46,33 ; 41,00$ and 38,08 t/ha respectively, with highly significant statistical differences, classifying themselves like forages. The Solapa, Pikis, Tabon, SR.90.328 and Toquecita clones had yields of 32,$58 ; 22,33 ; 20,75 ; 18,58$ and 16,50 t/ha of eatable roots respectively without statistical difference. The yields of eatable forage and roots obtained can be considered as successful and they allow preliminarily to conclude the different answer of production and promissory adaptation of the clones of sweet potato forage and double intention to the conditions of QuirozPiura Valley. It is suggested to continue with the test at other times.

\section{INTRODUCCIÓN}

El camote (Pomoea batatas Lam) es uno de los cultivos tradicionales más antiguos en el país. Se cultiva principalmente en los valles de costa y selva para producción de raíces reservantes (camote).

El cultivo de camote forrajero y de doble propósito es un cultivo nuevo en la zona y la región, debido a la inexistencia total de este tipo de cultivo, y merece favorables comentarios de los agricultores y ganaderos que requieren alimentos y forraje en forma permanente.

A mediados del 2001, fue introducido al Valle Quiroz, por CIDA PERÚ, iniciando experimentos exploratorios en parcelas de agricultores con buenos rendimientos de forraje y raíces reservantes de clones promisorios, no obstante las limitaciones presupuestales, logísticas y del escepticismo generalizado de los productores. El 2005 culminaron las investigaciones con el establecimiento de parcelas de comprobación y validación económica del clon DLP-2462 lactogénico, en comparación con el cv. local Solapa.

La actividad agropecuaria en el valle Quiroz y en especial de la Asociación de Agricultores y Ganaderos Santa Ana, se caracteriza por ser una explotación agrícola-pecuaria. Actualmente, la cédula de cultivos que se practica en el ámbito está formada por 20 cultivos entre transitorios y permanentes, siendo los más importantes el arroz (39\%), maíz amarillo duro (33\%), maní (17\%) y 11 $\%$ otros cultivos.

El cultivo de camote tradicional se halla relegado como cultivo secundario de autoconsu- mo, bajo el argumento de que (a) tiene "poca salida y bajos precios", y (b) la "semilla ya no produce bien", lo que podría ser la consecuencia directa de la prácticamente nula renovación de los cultivares locales, que son para producción de camote comestible principalmente para autoconsumo y de mínima utilización como forraje para el ganado.

Los agricultores del valle Quiroz acostumbran decidir la siembra de camote considerando principalmente sus necesidades de autoconsumo, disponibilidad de terreno y agua de riego. Tienen épocas clásicas de siembra que son los meses de abril y mayo, soslayando la situación de los precios y condiciones del mercado local, principalmente en la en ciudad de La Tina - Perú, zona de frontera con el Ecuador.

La situación expuesta en los puntos (a) y (b) preocupa a los productores, por lo que se interesan por renovar sus cultivares tradicionales por nuevos cultivares de mayores rendimientos, principalmente de raíces reservantes (camote) para alimentación humana, siendo el camote uno de los alimentos básicos de la dieta diaria familiar.

La ocurrencia del Fenómeno del Niño en los años 1983/84 y 1997/98 ha ocasionado pérdidas de cultivares locales como "saucecito", "morado", "pierna de señora", "pico de soña”, "pedrito", debido a la susceptibilidad de estos cultivares a factores patológicos y mezclas físicas, originando la disminución de la capacidad productiva con la consecuente pérdida de la calidad comercial.

La población ganadera en Piura según el III CENAGRO 1994 fue de 1050854 cabezas y estuvo conformada por $37 \%, 24 \%, 23 \%, 15 \%$ y $1 \%$ de caprinos, ovinos, vacunos, porcinos y equi- 
nos respectivamente. Gran parte de esta población se cría en el sistema de crianza extensiva con mínimo manejo técnico.

La disponibilidad de forrajes cultivados en la zona y región en general es muy escasa y no existe una cultura de producción y uso de forrajes cultivados, es decir, la ganadería se practica en función de la disponibilidad de pasturas naturales, residuos de cosechas o vegetación marginal.

En consecuencia, la alimentación del ganado es deficiente y no llega a cubrir los requerimientos nutricionales mínimos para su mantenimiento y mucho menos sus requerimientos para producción, más aún si sus requerimientos se incrementan en $25 \%$ en condiciones de libre pastoreo. Ante esta situación, se requiere tener nuevas alternativas forrajeras, de bajos costos y de sostenida y fácil producción.

En este aspecto, destaca el clon DLP2462 de aptitud forrajera, que presenta gran adaptación a la agricultura en agroecosistemas de los valles de la región y buena calidad nutricional. Sin embargo, antes de optar por esta alternativa forrajera es imprescindible conocer sus cualidades agronómicas, nutricionales (composición nutricional, palatabilidad y digestibilidad) y económicas (rentabilidad).

La situación expuesta hace imperativas la introducción y adaptación de nuevos cultivares de superiores cualidades, buenas características agronómicas, alto potencial de rendimiento de forraje (para el ganado caprino principalmente) y raíces reservantes, estabilidad de comportamiento en el tiempo y resistencia o tolerancia al ataque de plagas, y calidad comercial y culinaria para la renovación de los cultivares tradicionales.

El objetivo de la propuesta es presentar el plan de investigación realizado para la obtención del nuevo cultivar lactogénico y los resultados en términos agronómicos, nutricionales y económicos que sustentan su liberación.

\section{Origen del nuevo cultivar}

El clon DLP-2462 es originario del Perú, y constituye una de las accesiones del Banco de Germoplasma Mundial de Camote que mantiene en Lima Perú el Centro Internacional de la Papa - CIP, institución que proporcionó plantas madres libres de virus al Centro de Investigación y Desarrollo Agrario del Peru - CIDA PERÚ con Carta de envío PS-01-067, 07/agosto/2001.

La data de Colección, Caracterización y Reacción de factores bióticos y abióticos del clon DLP-2462 determinado por el Centro Internacional de la Papa - CIP, se detalla en el Anexo 1.

\section{Características}

Se menciona las características fenotípicas resaltantes observadas durante el Plan Experimental ejecutado en las condiciones agroecológicas del valle Quiroz - Piura.

\section{Planta}

- Tipo de crecimiento : Vigoroso

- Grosor de tallos : Mediano a grueso

- Color de tallos : Rojo vinoso

- Forma de la hoja : Cordada.

- Altura de planta : : 0,40 m en promedio.

- Hábito de crecimiento : Postrado muy extendido

- Color de nervaduras : Vinoso en el envés

- Período vegetativo : 140 a 160 días.

- Esquejes - semilla /planta

- Longitud tallo principal : 4,20 m.

- Materia seca $\quad: 18,2 \%$

- Rendimiento de follaje : 40,0 t/ha.

\section{Raíces reservantes}

- Forma

- Color de piel

: Alargada-elíptica

- Color de pulpa

Rosado vinoso

- Superficie de piel

: Amarilla

- Tipo de ojos

- Raíces reservantes

/planta

: Uniforme

: Superficiales

- Formación de raíces

- Materia seca

3 a 8

- Raíces comerciales : $40 \%$

- Raíces no comerciales: 60 \%

\section{Adaptación}

El clon DLP-2462 se adapta muy bien a las condiciones agroecológicas del valle Quiroz, valles interandinos de Piura y Costa Norte del país.

Comportamiento al ataque de plagas.

El clon DLP-2462 desarrolla un Índice de 
Área Foliar (IAF) de 2.26, que le permite tolerar las pérdidas de área foliar (agujeros en hojas) por ataque de plagas del follaje y lo hace poco vulnerable, performance observada en el valle Quiroz durante los 5 años (2001-2005) de evaluaciones agronómicas.

Los cortes oportunos de forraje y la cosecha a los 150 o 160 días después de la siembra, permiten lograr forraje y raíces reservantes prácticamente libres de plagas, principalmente del endémico gorgojo del camote Euscepes postfiaciatus. No se ha observado síntomas del ataque de nematodos en follaje y raíces reservantes.

\section{MATERIAL Y METODO}

Material genético experimental.

El material genético experimental detallado en el Tabla 1 estuvo compuesto por un grupo de 20 clones selectos de camote de aptitud forrajera y de doble propósito proveniente del Banco de Germoplasma Mundial de Camote del Centro Internacional de la Papa - CIP Lima y 3 cultivares nativos donados por los agricultores.

\section{Zona agroecológica experimental}

Las características de la zona agroecológica donde se realizaron los experimentos es la formación Bosque Seco Tropical, frecuentemente denominada Trópico Seco, entre 400 y 450 metros de altitud. Los suelos son de origen aluvial, textura principalmente franco a franco-arcillosa, $\mathrm{pH}$ ligeramente alcalino, buen drenaje donde discurren las aguas del río Quiroz. Los experimentos se realizaron en el ámbito de la Asociación de Agricultores y Ganaderos Santa Ana de Quiroz, en los sectores Santa Cruz, Santa Ana, El Torno y localidades de Puente Quiroz y San Joaquín.

\section{Tipo de productores colaboradores}

Los colaboradores fueron pequeños productores agrícola-ganaderos con experiencia en

Tabla 1. Material genético experimental

\begin{tabular}{cccc}
\hline Orden & CIP number & Collector number & Clones \\
\hline 1 & 420386 & ARB-158 & Acaro \\
2 & 420439 & ARB-265 & Calabacito \\
3 & 420476 & ARB-389 & Yema de Huevo \\
4 & 420120 & ARB-394 & Pikis \\
5 & 420068 & ARBUNAP-55 & Helena \\
6 & 420152 & ARBUNAP-74 & Kumala \\
7 & 440034 & BDI-Mohc & Mohc \\
8 & 401466 & CC-89 .213 & CC-89.213 \\
9 & 420713 & DLP-1308 & Morado \\
10 & 420248 & DLP-2448 & Dulce \\
11 & 420250 & DLP-2462 & DLP-2462 \\
12 & 420855 & DLP-2481 & DLP-2481 \\
13 & 420169 & DLP-275a & Tabón \\
14 & 420967 & DLP-3525 & unknown \\
15 & 421383 & DLP-3548 & DLP-3548 \\
16 & --- & DLP-90052 & DLP-90052 \\
17 & 421083 & RCB-IN-5 & Tipo.3 \\
18 & 440045 & SPV-55 & DLP-2462 \\
19 & 440049 & SPV-65 & Mojave \\
20 & 190023.59 & SR-90.323 & SR-90.323 \\
21 & --- & SOLAPA & Solapa-T1 \\
22 & --- & SANTOS & Santos-T2 \\
23 & --- & PICO DE SONA & Pico de Soña-T3 \\
\hline & & &
\end{tabular}


el cultivo de camote común y crianza de caprinos criollos, a quienes se motivó y organizó para su participación en las acciones de Investigación Participativa y Capacitación Tecnológica con la metodología de Escuelas de Campo para Agricultores - ECA's, llevándose a cabo los experimentos y evaluaciones conjuntas y así asegurar la adopción del clon y la moderna tecnología de producción.

Plan experimental y metodología de capacitación

El Plan Experimental de Investigación Participativa consistió en la ejecución de experimentos o ensayos en parcelas de pequeños productores tipificados en el ítem 4.1.3. La Unidad Experimental estuvo conformada generalmente por 4 surcos donde se diseñaron las franjas de corte de forraje A y $B$ detallado en el grafico 1. gica final. Con los resultados experimentales se procedió a los Análisis Estadísticos ANVA y Prueba de Dunnett de los clones mejorados (tratamientos) comparados con los clones testigo.

Paralelamente, se desarrollaron acciones de Capacitación Tecnológica a los productores colaboradores en aplicación de la Metodología de Escuelas de Campo para Agricultores (ECA's), organizadas en tres escuelas en forma progresiva y acorde a la evolución fenotípica de los experimentos en los diferentes sectores; constituyéndose las Parcelas Experimentales en lugar y material de capacitación tecnológica.

\section{Criterios de evaluación}

Los criterios de evaluación fueron: (i) Criterios de los Agricultores, referidos al tamaño de

Gráfico 1. Diseño experimental Strip plot (parcela en franjas).

Detalle de la unidad experimental tipo

\begin{tabular}{|c|c|c|c|c|}
\hline A & $\begin{array}{l}\text { surco } 1 \\
\text { Corte A1: } 75 \mathrm{dds} \\
\text { Corte A2: } 75 \mathrm{dds}\end{array}$ & surco 2 & surco $3 \quad$ surco 4 & L.1 (m) \\
\hline B & $\begin{array}{c}\text { Corte B1: } 80 / 90 \mathrm{dds} \\
\text { Corte B2: } 70 / 60 \mathrm{dds} \\
\text { L. } 2(\mathrm{~m})\end{array}$ & & $\longrightarrow 1,0 \mathrm{~m} \leftarrow$ & \\
\hline
\end{tabular}

Área : L. $1 \times$ L. $2=\mathrm{m}^{2}$

Follaje : Rdto A = Corte A1 + Corte A2. / Rdto B: corte B1 + corte B2.

Raices : Rdto A = Raíces A / Rdto B = Raices B.

La complejidad y el rigor científico de los ensayos fueron de menor a mayor rigurosidad, conducentes al logro de los objetivos, detalle en Gráfico 2.

Los experimentos planeados fueron:

1. Ensayos Intermedios, en Diseño Estadístico STRIP PLOT (parcela en franjas), con arreglo en $B C R / 4 r$.

2. Ensayos Avanzados; en Diseño Estadístico STRIP-PLOT (parcela en franjas), con arreglo en $B C R / 4 r$.

3. Parcelas de Comprobación; en Diseño Estadístico STRIP PLOT (parcela en franjas), con arreglo en $\mathrm{BCR} / 4 \mathrm{r}$.

4. Parcelas Demostrativas; en campos semicomerciales con fines de demostración tecnoló- las raíces comerciales, y calidad culinaria por su costumbre de producir siempre camote tradicional; y (ii) Criterios del Equipo Técnico, relacionados a las épocas de corte y rendimiento de follaje fresco, raíces reservantes, tamaño y uniformidad de las raíces comerciales, sanidad y calidad culinaria.

Los experimentos y evaluaciones fueron realizados en forma conjunta entre el Equipo Técnico investigador y los agricultores colaboradores previamente capacitados. El Equipo Técnico Investigador, adicionalmente, realizó evaluaciones de los parámetros fenológicos sobre vigor, uniformidad y cobertura (VUC) de plantas, épocas de corte y rendimiento ( $\mathrm{t} / \mathrm{ha}$ ) de follaje fresco, rendimiento y clasificación de la raíces reservantes (t/ ha), evaluación del ataque por plagas, pudrición y 
Gráfico 2. Plan de investigación en camote forrajero.

\begin{tabular}{|c|c|c|c|c|}
\hline Años & Clones & Tipo ensayo & Evaluaciones & Responsable \\
\hline $2001-2003$ & $20+2$ & Ensayos intermedios & Biológicas & Cida Perú+agricola \\
\hline $2004 \mathrm{a}$ & $20+2$ & Ensayos avanzados & Biológicas+económicas & Py. Camote forrajero \\
\hline $2004 \mathrm{~b}$ & $4+1$ & Ensayos avanzados & Biológicas+económicas & Py. Camote forrajero \\
\hline 2005 & $2+1$ & Parcelas comprobación & Económicas+biológicas & Py. Camote Forrajero \\
\hline 2006 & $2+1$ & Parcelas demostrativas & Demostrativas & Py. Camote Forrajero \\
\hline & & & Cv. $\rightarrow$ DLP-2462 & Doble propósito \\
\hline
\end{tabular}

craking o rajaduras (PPC) de raíces reservantes, número y peso de raíces comerciales y no comerciales y determinación del porcentaje de materia seca en muestras de follaje y raíces reservantes, previo secado al medio ambiente y pesado en balanza mecánica y electrónica.

\section{Ejecución del plan experimental}

El material genético utilizado en los experimentos contaba con data previa de colección, evaluaciones de identificación, caracterización y experimentos preliminares (anexo 1.) realizados por el Centro Internacional de la Papa-CIP, en sus estaciones de Selva Baja (Yurimaguas), Selva Alta (San Ramón) y Costa Central (Cañete, Huaral y Ate). El material genético recepcionado se encontraba en esa etapa de evaluación.

Los experimentos sucesivos en el valle Quiroz fueron realizados en pequeñas parcelas de agricultores, inicialmente por CIDA PERÚ, en ejecución de su Plan Experimental 2001-2003 y seguidamente en el marco del Plan Experimental 2004-2006 del Proyecto Camote Forrajero, ambos planes agrupados en:

\section{Experimentos intermedios (EI) 2001-2003}

Este grupo de experimentos fue realizado en el marco del Plan Experimental 2001-2003 (propio de CIDA PERÚ), en Diseño Bloque Completo al Azar, con 4 repeticiones en dos periodos de cultivo. Se evaluó los rendimientos de forraje y raíces reservantes de 20 clones mejorados introducidos y 02 clones-testigo locales más difundidos en la zona (tratamientos en estudio), y no se establecieron metas previas de rendimientos.

\section{Experimentos avanzados (EA) 2004}

Este grupo de experimentos fue realizado en el marco del Proyecto Camote Forrajero (Contrato 018-2003/FTA/INCAGRO), y estuvo conformado por clones mejorados (tratamientos) procedentes de Experimentos Intermedios; sembrados en tres localidades. El periodo vegetativo de los experimentos se estandarizó en 150 días. Las metas de rendimientos fueron de 40,0 y 15,0 t/ha, para follaje y raíces reservantes respectivamente.

El diseño experimental estándar fue Strip Plot (parcela en franjas) con arreglo en Bloque Completo al Azar y 4 repeticiones. Para la cosecha de follaje, las unidades experimentales se dividieron en franjas $A$ y $B$; $y$ se hizo cortes $A 1$, a 75 días y cortes $\mathrm{B} 1$ a 80 ó 90 días, y cortes $\mathrm{A} 2$ y B2 a los 75 ó 80 días después (150-160 días en total), en siembras sucesivas y épocas diferentes durante el año.

\section{Parcelas de comprobación (PC) 2005}

Este grupo fue instalado en parcelas semicomerciales; para los cortes de follaje las unidades experimentales se dividieron en franjas parte $A($ cortes A1 + A2) y parte B (cortes B1 + B2). La cosecha de raíces reservantes se realizó durante el último corte de follaje (cortes A2 y B2), obteniéndose así los rendimientos de forraje y raíces reservantes de los tratamientos.

En los tres grupos los resultados experi- 
mentales se sometieron a los Análisis Estadísticos ANVA y Prueba de Dunnett de los clones mejorados con los clones testigos (tratamientos).

En las parcelas de comprobación se evaluaron los rendimientos de forraje y raíces reservantes de los dos (2) mejores clones mejorados (tratamientos) y el clon Solapa-testigo, más los análisis económicos y de rentabilidad.

\section{Parcelas demostrativas (PD) 2006}

Estas parcelas fueron cultivos semicomerciales y constituyeron básicamente material para acciones demostrativas a los colaboradores y productores en general y también para las demostraciones durante el Día de Campo programado para la Liberación de los nuevos cultivares logrados con el Proyecto. La data generada por su naturaleza no es parte del presente informe.

\section{Pruebas de palatabilidad y digestibilidad}

Para determinar las características organolépticas y calidad del forraje, en la etapa de Parcelas de Comprobación, se hizo análisis de laboratorio a fin de cuantificar la composición nutricional (porcentual) y pruebas de palatabilidad y digestibilidad del clon DLP-2462 y cv. Solapa, en caprinos criollos del valle Quiroz.

\section{Determinación de materia seca de follaje y raí ces reservantes}

El camote forrajero como insumo para alimentación animal se valoriza mejor como forraje seco (y no solo como forraje fresco), procediéndose a determinar el contenido porcentual de materia seca en follaje (hojas y tallos) y raíces reservantes (camote), ambos como fuentes calórico-proteicas para alimento humano o animal.

\section{Composición nutricional del follaje y raíces re- servantes}

La determinación de sus características organolépticas y composición nutricional, se hizo para su utilización como insumo en programas de alimentación humana y animal (fresco o procesado) o para transformación en harinas, almidones y alcoholes, insumo para alimentos preparados de canes o usos en la fabricación de autopartes biodegradables de vehículos o biodiésel ecológico.

\section{RESULTADO Y DISCUCIÓN}

\section{Evaluaciones agronómicas}

\section{Experimentos intermedios (EI) 2001-2003}

Los Experimentos Intermedios El-101 y El-103 fueron conducidos en el sector Puente Quiroz, donde se evaluó la producción de follaje y raíces reservantes; por la característica exploratoria de estos experimentos no se establecieron metas previas de rendimientos. En la conducción de estos experimentos se combinaron dos tecnologías, la Tecnología Local ${ }^{2}$ de producción (en "torromotos", "camellón" o "hueco") de camote común de los agricultores y la Tecnología Nueva (en surcos con prácticas intensivas) para producción de forraje y raíces reservantes (camote). El objetivo era generar una Tecnología Moderna de Producción de Forraje y Raíces Reservantes adaptada a las condiciones locales. En la tabla 2, se resumen los rendimientos.

Experimento El-101, conducido en el periodo primavera-verano-otoño, en 230 días de cultivo.

1. Los rendimientos de follaje fluctúan entre 26,7 y 16,2 t/ha, sin diferencias estadísticas entre los clones (tratamientos). En el cultivo hubo fuerte defoliación debido al largo periodo vegetativo; destaca el clon DLP-2462 con el máximo rendimiento de $26,7 \mathrm{t} / \mathrm{ha}$ de follaje.

2. Los rendimientos de raíces reservantes fluctúan entre 54,7 y 3,4 t/ha, DLP-2462 produjo $7.6 \mathrm{t} / \mathrm{ha}$.; existiendo diferencias estadísticas significativas, la Prueba de Dunnett indica que el clon Toquecita supera todos los clones.

3. Todos los clones, debido al largo periodo de cultivo (230 días), producen raíces reservantes, a excepción de los clones Calabacito y DLP-3548, que no producen raíces reservantes.

Experimento El-103 conducido en el periodo verano-otoño, en 190 días de cultivo.

4. Los rendimientos de follaje fluctúan entre 44,4 y $16,5 \mathrm{t} / \mathrm{ha}$, en promedio. Estadísticamente todos los clones tienen rendimientos similares a excepción del clon Pikis, que alcanza un rendimiento estadísticamente inferior a Solapa-testigo.

5. Los rendimientos de raíces reservantes fluctúan entre 53,4 y 0,5 t/ha, en promedio, des- 
Tabla 2. Resúmen de rendimientos de follaje y raíces reservantes (t/ha).

\begin{tabular}{|c|c|c|c|c|c|c|c|c|c|c|}
\hline \multirow{2}{*}{ Orden } & \multirow{2}{*}{$\begin{array}{l}\text { Collector } \\
\text { number }\end{array}$} & \multirow{2}{*}{ Clones } & \multicolumn{4}{|c|}{$\begin{array}{c}\text { Exp. : EI-101 } \\
\text { PrimaveraVeranoOtoño }\end{array}$} & \multicolumn{4}{|c|}{$\begin{array}{l}\text { Exp. : EI-103 } \\
\text { VeranoOtoño }\end{array}$} \\
\hline & & & Follaje & $\begin{array}{l}\text { Dunnett } \\
\mathrm{P}<.05\end{array}$ & Raíces & $\begin{array}{l}\text { Dunnett } \\
\mathrm{P}<.05\end{array}$ & Follaje & $\begin{array}{l}\text { Dunnett } \\
\mathrm{P}<.05\end{array}$ & Raíces & $\begin{array}{c}\text { Dunnett } \\
\mathrm{P}<.05\end{array}$ \\
\hline 1 & DLP-2462 & DLP-2462 & 26,7 & ns & 7,6 & ns & 44,4 & ns & 3,9 & ns \\
\hline 2 & RCB-IN-5 & Tipo.3 & 22,5 & ns & 3,4 & $\mathrm{~ns}$ & 27,2 & $\mathrm{~ns}$ & 4,6 & ns \\
\hline 3 & BDI-Mohc & Mohc & 22,4 & ns & 38,6 & $\mathrm{~ns}$ & 27,7 & $\mathrm{~ns}$ & 13,9 & ns \\
\hline 4 & SPV-55 & DLP-2462 & 22,2 & ns & 54,7 & $*$ & 19,2 & $\mathrm{~ns}$ & 53,4 & $*$ \\
\hline 5 & ARBUNAP-55 & Helena & 22,2 & ns & 7,0 & ns & 22,4 & ns & 0,0 & ns \\
\hline 6 & SR-90.323 & SR-90.323 & 21,8 & ns & 36,5 & ns & 25,0 & ns & 13,2 & ns \\
\hline 7 & CC-89.213 & CC.89.213 & 21,8 & ns & 26,5 & ns & 18,2 & ns & 6,6 & ns \\
\hline 8 & DLP-3525 & Unknown & 20,8 & ns & 23,6 & ns & 33,4 & $\mathrm{~ns}$ & 1,9 & ns \\
\hline 9 & ARB-265 & Calabacito & 20,8 & ns & 0,0 & $*$ & 24,4 & ns & 0,0 & ns \\
\hline 10 & ARB-394 & Pikis & 20,5 & ns & 18,8 & ns & 16,5 & $*$ & 1,0 & ns \\
\hline 11 & ARB-389 & Yema de $\mathrm{H}$. & 20,4 & ns & 15,0 & ns & 20,8 & ns & 5,6 & ns \\
\hline 12 & ARBUNAP-74 & Kumala & 19,1 & ns & 13,3 & ns & 26,4 & ns & 2,5 & ns \\
\hline 13 & DLP-275a & Tabón & 18,8 & $\mathrm{~ns}$ & 52,8 & ns & 25,2 & ns & 20,1 & ns \\
\hline 14 & ----- & Pico Soña & 18,7 & test & 27,2 & test & 20,1 & ns & 7,2 & ns \\
\hline 15 & DLP-90052 & DLP90052 & 18,6 & $\mathrm{~ns}$ & 5,4 & $\mathrm{~ns}$ & 30,3 & ns & 0,9 & ns \\
\hline 16 & DLP-3548 & DLP-3548 & 18,1 & ns & 0,0 & $*$ & 30,2 & ns & 0,8 & ns \\
\hline 17 & ARB-158 & Acaro & 17,2 & ns & 3,4 & ns & 27,2 & $\mathrm{~ns}$ & 0,0 & ns \\
\hline 18 & DLP-2481 & DLP-2481 & 16,9 & ns & 3,4 & ns & 27,6 & ns & 5,7 & ns \\
\hline 19 & DLP-2448 & Dulce & 16,2 & ns & 14,2 & ns & 28,6 & $\mathrm{~ns}$ & 0,0 & ns \\
\hline 20 & DLP-1308 & Morado & 16,2 & ns & 8,5 & ns & 30,4 & ns & 1,6 & ns \\
\hline 21 & SPV-65 & Mojave & ---- & ---- & ---- & ---- & 26,2 & $\mathrm{~ns}$ & 0,5 & ns \\
\hline \multirow[t]{4}{*}{22} & ----- & Solapa & ---- & ---- & ---- & ---- & 36,3 & test & 10,9 & test \\
\hline & & Meta & n.d & & n.d & & n.d & & n.d & \\
\hline & & Promedio & 20,1 & & 18,0 & & 26,7 & & 7,0 & \\
\hline & & c.v. $\%$ & 22,1 & & 52,3 & & 29,1 & & 121,5 & \\
\hline
\end{tabular}

n.d. $=$ no determinado $/ \mathrm{ns}=$ no significativo $/ *=$ significativo

tacando Toquecita con 53.4 t/ha. Los clones Helena, Dulce, Acaro y Calabacito no producen raíces reservantes.

6. En los dos experimentos destaca el Clon DLP2462, con los mayores rendimientos de follaje de 26,7 y 44,4 t/ha, mostrando su aptitud forrajera.

7. Los clones DLP-2462 y Toquecita destacan como clones promisorios por sus altos rendimientos de follaje y raíces reservantes, como clones de aptitud forrajera y doble propósito respectivamente. Esta información sirve de base para pasar al siguiente grupo de Experimentos Avanzados.

\section{Experimentos avanzados (ea) 2004}

Este grupo de Experimentos Avanzados EA-104, EA-204 y EA-404 fue conducido en dos localidades y tres periodos diferentes de cultivo. Los rendimientos son muy variables. En las tablas
3 y 4 se resumen los rendimientos de follaje y raíces reservantes respectivamente.

\section{Experimento EA-104, localidad Santa Cruz.}

1. Los rendimientos de follaje fluctúan entre 39,7 y $20.1 \mathrm{t} / \mathrm{ha}$, siendo similares estadísticamente, a excepción Yema de Huevo y Pikis que alcanzan rendimientos estadísticamente menores a Solapa.

2. El mayor rendimiento de follaje lo alcanza el clon Acaro con 39,7 t/ha, y DLP-2462, ocupa el 3er lugar con $36,8 \mathrm{t} / \mathrm{ha}$. En este ensayo ninguno de los clones supera la meta de 40,0 t/ha.

Experimento EA-204, localidad El Torno.

3. Los rendimientos de follaje fluctúan entre 56,1 y $16,7 \mathrm{t} / \mathrm{ha}$, siendo estadísticamente diferentes; los cultivares locales Solapa y Santos 
Tabla 3. Resúmen de rendimientos de follaje (t/ha).

\begin{tabular}{|c|c|c|c|c|c|c|c|c|}
\hline \multirow{2}{*}{ Orden } & \multirow{2}{*}{$\begin{array}{l}\text { Collector } \\
\text { number }\end{array}$} & \multirow{2}{*}{ Clones } & \multicolumn{2}{|c|}{$\begin{array}{l}\text { Exp. EA-104 } \\
\text { Verano-Otoño }\end{array}$} & \multicolumn{2}{|c|}{$\begin{array}{l}\text { Exp. EA-204 } \\
\text { OtoñoInvierno }\end{array}$} & \multicolumn{2}{|c|}{$\begin{array}{c}\text { EA-404 } \\
\text { VeranoOtoño }\end{array}$} \\
\hline & & & Follaje & $\begin{array}{c}\text { Dunnett } \\
\mathrm{P}<.05\end{array}$ & Follaje & $\begin{array}{c}\text { Dunnett } \\
\mathrm{P}<.05\end{array}$ & Follaje & $\begin{array}{c}\text { Dunnett } \\
\mathrm{P}<.05\end{array}$ \\
\hline 1 & ARB-158 & Acaro & 39,7 & $\mathrm{~ns}$ & 42,1 & $*$ & 34,5 & $\mathrm{~ns}$ \\
\hline 2 & DLP-3525 & Unknown & 38,2 & ns & 36,8 & $*$ & 38,7 & ns \\
\hline 3 & DLP-2462 & DLP-2462 & 36,8 & ns & 45,3 & ns & 41,3 & ns \\
\hline 4 & DLP-2448 & Dulce & 34,8 & ns & 48,1 & ns & 44,6 & $\mathrm{~ns}$ \\
\hline 5 & ARBUNAP-74 & Kumala & 34,2 & ns & 44,6 & ns & 44,6 & $\mathrm{~ns}$ \\
\hline 6 & BDI Mohc & Mohc & 33,9 & ns & 47,6 & ns & 53,3 & ns \\
\hline 7 & DLP-1308 & Morado & 33,7 & ns & 33,0 & $*$ & 37,4 & ns \\
\hline 8 & RCB-IN-5 & Tipo. 3 & 30,9 & ns & 32,2 & $*$ & 29,4 & $*$ \\
\hline 9 & ARBUNAP-55 & Helena & 30,3 & ns & 33,5 & $*$ & 32,5 & $\mathrm{~ns}$ \\
\hline 10 & DLP-3548 & DLP-3548 & 29,8 & ns & 47,0 & ns & 36,5 & ns \\
\hline 11 & SR-90323 & SR-90323 & 29,7 & ns & 40,3 & $*$ & 33,5 & $\mathrm{~ns}$ \\
\hline 12 & SPV-65 & Mojave & 28,8 & ns & 49,8 & ns & 33,8 & ns \\
\hline 13 & CC. 89.213 & CC. 89.213 & 28,3 & ns & 25,5 & $*$ & 36,6 & ns \\
\hline 14 & DLP-275a & Tabón & 28,1 & ns & 27,9 & $*$ & 24,5 & $*$ \\
\hline 15 & SPV-55 & Toquecita & 27,3 & ns & 20,8 & $*$ & 38,5 & ns \\
\hline 16 & ARB-265 & Calabacito & 26,5 & ns & 51,4 & ns & 38,5 & ns \\
\hline 17 & DLP-90052 & DLP-90052 & 26,4 & ns & 49,8 & ns & 20,6 & $*$ \\
\hline 18 & ----- & Santos-T2 & 25,4 & ns & 16,7 & $*$ & --- & --- \\
\hline 19 & ARB-389 & Yema de $\mathrm{H}$. & 21,7 & $*$ & 23,8 & $*$ & 24,0 & $*$ \\
\hline 20 & ARB-394 & Pikis & 20,1 & $*$ & 37,7 & $*$ & 35,8 & ns \\
\hline \multirow[t]{4}{*}{21} & ----- & Solapa-T1 & 38,9 & test & 56,1 & test & 42,3 & test \\
\hline & Meta & & 40,0 & & 40,0 & & 40,0 & \\
\hline & Promedio & & 30,6 & & 38,6 & & 36,0 & \\
\hline & C.V. $\%$ & & 29,09 & & 22,86 & & 23,11 & \\
\hline
\end{tabular}

$\mathrm{ns}=$ no significativo $/ *=$ significativo

ocupan el primer y último lugar, demostrando su aptitud forrajera y raicera respectivamente.

4. El mayor rendimiento de follaje lo alcanza el Solapa-T1 con 56,1 t/ha, y el DLP-2462 produce $45,3 \mathrm{t} / \mathrm{ha}$, ocupando el $8 \mathrm{vo}$ lugar, siendo los rendimientos estadísticamente similares. En este ensayo, once (11) clones superan la meta de 40,0 t/ha.

Experimento EA-404, localidad EI Torno.

5. Los rendimientos de follaje fluctúan entre 53,3 y 20,6 t/ha, existiendo diferencias estadísticas entre los rendimientos. Destaca el rendimiento del clon DLP-2462 que logra 41,3 t/ha y ocupa el 5 to lugar. En este ensayo, cinco (5) clones superan la meta de 40,0 t/ha.

6. En todos los casos, los rendimientos de follaje proceden de 2 cortes, no existiendo diferencias estadísticas; los primeros cortes A1 y B1, a 75 y 90 días después de la siembra; y los segundos cortes A2 y B2, a 75 y 60 días después del primer corte (150 días total) se detallan en el Gráfico 2.

Experimento EA-104, localidad Santa Cruz.

1. Los rendimientos de raíces reservantes fluctúan de 33,4 a 0,2 t/ha, y todas son estadísticamente diferentes, a excepción del Mohc, que produce similar rendimiento al clon Toquecita.

2. Toquecita, destaca nítidamente con 33,4 t/ha con el máximo rendimiento y le sigue Santos-

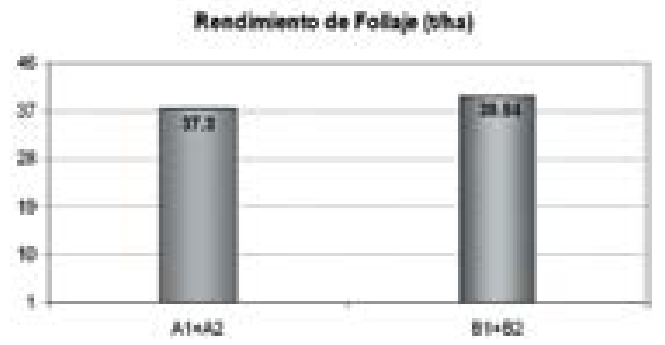

Gráfico 2. Experimento EA-204: Rendimiento de follaje con 2 cortes. 
Tabla 4. Resúmen de raíces reservantes (t/ha).

\begin{tabular}{|c|c|c|c|c|c|c|c|c|}
\hline \multirow{2}{*}{ Orden } & \multirow{2}{*}{$\begin{array}{l}\text { Collector } \\
\text { number }\end{array}$} & \multirow{2}{*}{ Clones } & \multicolumn{2}{|c|}{$\begin{array}{l}\text { Exp. EA-104 } \\
\text { VeranoOtoño }\end{array}$} & \multicolumn{2}{|c|}{$\begin{array}{l}\text { Exp. EA-104 } \\
\text { OtoñoInvierno }\end{array}$} & \multicolumn{2}{|c|}{$\begin{array}{l}\text { Exp. EA-104 } \\
\text { VeranoOtoño }\end{array}$} \\
\hline & & & Follaje & $\begin{array}{c}\text { Dunnett } \\
\mathrm{P}<.05\end{array}$ & Follaje & $\begin{array}{c}\text { Dunnett } \\
\mathrm{P}<.05\end{array}$ & Follaje & $\begin{array}{c}\text { Dunnett } \\
\mathrm{P}<.05\end{array}$ \\
\hline 1 & SPV-55 & Toquecita & 33,4 & $*$ & 34,0 & $*$ & 18,2 & $*$ \\
\hline 2 & ------ & Santos-T2 & 16,7 & test & 12,9 & test & ---- & ---- \\
\hline 3 & BDI Mohc & Mohc & 14,4 & ns & 22,5 & $*$ & 9,7 & $*$ \\
\hline 4 & SR-90.323 & SR-90.323 & 6,5 & $*$ & 13,5 & $\mathrm{~ns}$ & 8,4 & $*$ \\
\hline 5 & DLP-275a & Tabón & 5,4 & * & 9,9 & $\mathrm{~ns}$ & 5,4 & ns \\
\hline 6 & DLP-3525 & Unknown & 5,2 & * & 4,7 & $*$ & 0,2 & $\mathrm{~ns}$ \\
\hline 7 & CC.89.213 & CC.89.213 & 3,5 & * & 11,1 & ns & 6,3 & $*$ \\
\hline 8 & ARB-389 & Yema de H. & 3,2 & $*$ & 4,0 & $*$ & 6,4 & $*$ \\
\hline 9 & ARBUNAP-74 & Kumala & 2,6 & * & 5,2 & $*$ & 1,9 & ns \\
\hline 10 & ARB-394 & Pikis & 1,4 & $*$ & 7,0 & $*$ & 4,4 & $\mathrm{~ns}$ \\
\hline 11 & DLP-1308 & Morado & 1,4 & $*$ & 0,4 & $*$ & 0,2 & $\mathrm{~ns}$ \\
\hline 12 & ARB-158 & Acaro & 1,0 & * & 0,1 & $*$ & 0,6 & ns \\
\hline 13 & DLP 2462 & DLP 2462 & 0,5 & $*$ & 1,5 & $*$ & 0,0 & ns \\
\hline 14 & DLP-90052 & DLP-90052 & 0,3 & $*$ & 0,7 & * & 1,3 & ns \\
\hline 15 & ARB-265 & Calabacito & 0,2 & $*$ & 0,4 & $*$ & 0,1 & ns \\
\hline 16 & ----- & Solapa-T1 & 0,2 & $*$ & 4,2 & $*$ & 1,7 & test \\
\hline 17 & DLP-2448 & Dulce & 0,0 & $*$ & 0,8 & $*$ & 0,8 & ns \\
\hline 18 & SPV-65 & Mojave & 0,0 & $*$ & 0,1 & $*$ & 0,1 & $\mathrm{~ns}$ \\
\hline 19 & DLP-3548 & DLP-3548 & 0,0 & $*$ & 0,2 & $*$ & 0,0 & $\mathrm{~ns}$ \\
\hline 20 & ARBUNAP-55 & Helena & 0,0 & $*$ & 1,1 & $*$ & 0,0 & $\mathrm{~ns}$ \\
\hline \multirow[t]{4}{*}{21} & RCB-IN-5 & Tipo. 3 & 0,0 & $*$ & 2,4 & $*$ & 1,4 & ns \\
\hline & Meta & & 15,0 & & 15,0 & & 15,0 & \\
\hline & Promedio & & 4,6 & & 6,5 & & 3,4 & \\
\hline & C.V. $\%$ & & 29,69 & & 35,45 & & 80,05 & \\
\hline
\end{tabular}

ns $=$ no significativo $/ *=$ significativo

T2 con 16,7 t/ha, y ambos superan la meta de 15,0 t/ha. DLP-2462 rinde 0,5 t/ha.

\section{Experimento EA-204, localidad EI Torno.}

3. Los rendimientos de raíces reservantes fluctúan de 34,0 a 0,1 t/ha, y son estadísticamente diferentes.

4. Toquecita, destaca nítidamente con 34,0 t/ha con el máximo rendimiento y le sigue Mohc con 22,5 t/ha, y ambos superan la meta de 15,0 t/ha. DLP-2462 logra 1,5 t/ha.

\section{Experimento EA-404, localidad EI Torno.}

5. Los rendimientos de raíces reservantes fluctúan de 18,2 a 0,1 t/ha, y son estadísticamente diferentes.

6. Toquecita, destaca nítidamente con $18,2 \mathrm{t} / \mathrm{ha}$ con el máximo rendimiento y solo este clon supera la meta de 15,0 t/ha; DLP-2462 no produjo raíces reservantes.
7. Los rendimientos de raíces reservantes son estadísticamente diferentes en los tres experimentos avanzados, destacando el clon Toquecita, con rendimientos de 33,$4 ; 34,0$ y 18,2 t/ha en los tres experimentos, siendo superior en 123,126 y $21 \%$ más que el rendimiento meta (15,0 t/ha); demostrando nítidamente su superioridad productiva de clon promisorio y clasificándose como un clon de doble propósito por excelencia.

8. El cultivar Solapa, ampliamente cultivado como productor de raíces reservantes, alcanzó rendimientos de 0,2, 4,2 y 1,7 t/ha, demostrando su condición de extremadamente tardío de 7 a 8 meses de periodo vegetativo para la producción de raíces reservantes. En este prolongado periodo de cultivo, las raíces reservantes son fuertemente atacados por el gorgojo del camote, plaga endémica que se encuentra en los suelos del valle Quiroz. 


\section{Parcelas de comprobación-validación (pc)} 2005.

Concluida la etapa de Experimentos Avanzados, se pasó a la siguiente etapa de Parcelas de Comprobación- Validación, las que fueron conducidas en dos localidades y dos épocas de cultivo diferentes para comprobar la perfomance del clon mejorado DLP-2462 comparado con el cultivar Solapa. Manteniéndose las metas de rendimientos de 40.0 y 15.0 t/ha, para follaje y raíces respectivamente. Tabla 5 . vidad. Ninguno de los clones supera la meta establecida de 15,0 t/ha.

En la PC-405 instalado en la localidad de San Joaquín durante el periodo Primavera-Verano.

3. Los rendimientos de follaje son de 52,7 y 59,5 t/ha, para el clon DLP-2462 y el cv Solapa; superando en 12,7 y 19,5 t/ha la meta establecida de 40,0 t/ha.

4. Los rendimientos de raíces reservantes son de 1,0 y 2,9 t/ha para DLP-2462 y Solapa,

Tabla 5. Resúmen de rendimientos de follaje y raíces reservantes ( $\mathrm{t} / \mathrm{ha})$.

\begin{tabular}{|l|c|c|c|c|c|c|c|c|}
\hline $\begin{array}{c}\text { Localidades } \\
\text { Clones }\end{array}$ & \multicolumn{2}{|c|}{$\begin{array}{c}\text { Exp.: PC-105 } \\
\text { OtoñoInvierno }\end{array}$} & \multicolumn{2}{c|}{$\begin{array}{c}\text { Exp.: PC-405 } \\
\text { PrimaveraVerano }\end{array}$} & \multicolumn{2}{c|}{ Follaje } & \multicolumn{2}{c|}{ Raíces } \\
\hline & Follaje & Raíces & Follaje & Raíces & Promedio & $\%$ & Promedio & $\%$ \\
\hline DLP-2462 & 36.1 & 2.9 & 52.7 & 1.0 & 44.4 & 92 & 2.0 & 39 \\
SOLAPA (T) & 37.5 & 7.2 & 59.5 & 2.9 & 48.5 & 100 & 5.1 & 100 \\
Promedio & 36.8 & 5.0 & 56.1 & 2.0 & --- & --- & --- & --- \\
Meta & 40.0 & 15.0 & 40.0 & 15.0 & 40.0 & --- & 15.0 & --- \\
\hline
\end{tabular}

En la PC-105, instalado en la localidad de El Torno durante el periodo Otoño-Invierno.

1. Los rendimientos de follaje son de 36,1 y 37,5 t/ha para DLP-2462 y Solapa respectivamente; ninguno de los clones supera la meta de $40,0 \mathrm{t} / \mathrm{ha}$ de follaje.

2. Los rendimientos de raíces reservantes son de 2,9 y 7,2 t/ha para DLP-2462 y Solapa respectivamente, notándose claramente que el clon DLP-2462 muestra su poca producti- notándose claramente que el clon DLP-2462 mantiene su baja productividad de raíces reservantes.

5. Los rendimientos promedio de follaje alcanzado por DLP-2462 y Solapa es de 44,4 y 48,5 t/ha para ambas localidades, superando la meta de 40,0 t/ha, ratificando ambos su aptitud forrajera.

6. Los rendimientos promedio de raíces reservantes son de 2,0 y 5,1 t/ha para DLP-2462 y Solapa respectivamente, es decir, DLP-2462 produce 1,5 veces menos que Solapa.

Gráfico 3. Evolución de rendimientos de follajes (t/ha): DLP-2462 vs Solapa

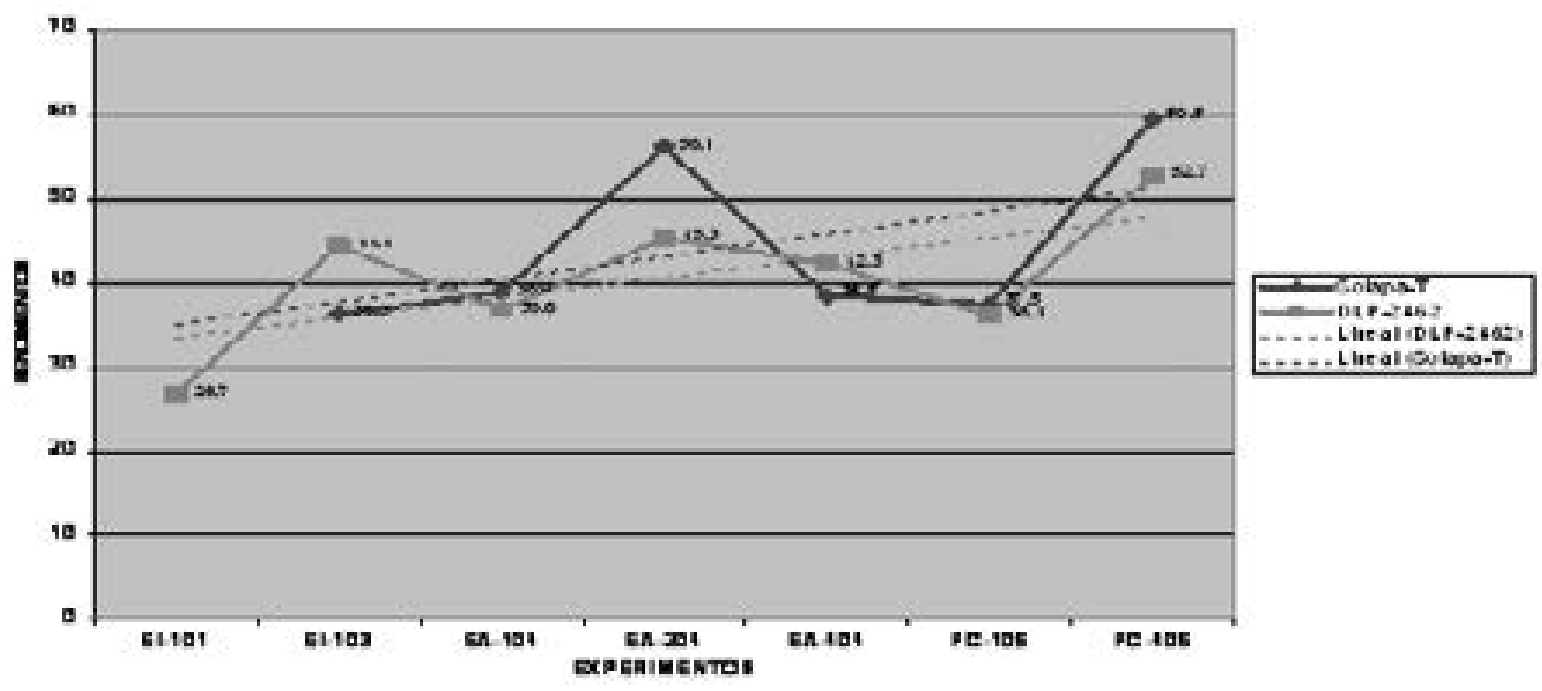




\section{Evolución de rendimientos de follaje y raíces reservantes}

En los gráficos 3 y 4 se muestran los resúmenes de la evolución de los rendimientos promedios comparativos del clon DLP-2462 y del cv. Solapa, en los tres grupos de experimentos. dencias son decrecientes para ambos.

5. Las disminución de los rendimientos de raíces reservantes del clon DLP-2462 y el cv. Solapa probablemente se debe a que a más veces de cultivo se produce mayor infestación de enfermedades, especialmente viróticas, consecuentemente, la producción disminuye.

\section{Gráfico 4. Evolución de rendimientos de raíces reservantes (t/ha): DLP-2462 vs Solapa}

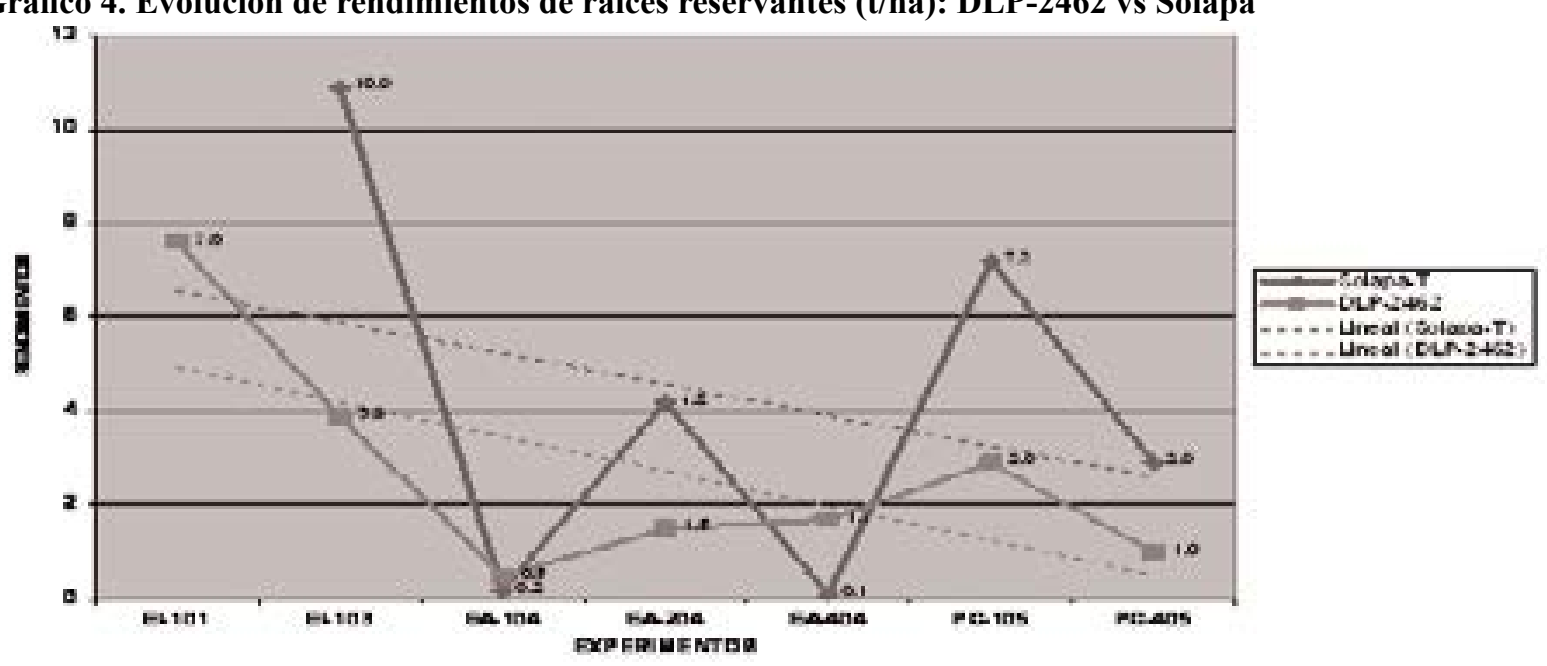

1. Los rendimientos promedios de follaje del clon DLP-2462 son menores a los cv. Solapa, debido a la reciente adaptación del clon DLP. 2462, frente a la performance del cv. Solapa.

2. Las tendencias de rendimientos de follaje del clon DLP-2462 y el cv. Solapa son crecientes y positiva, siendo la variabilidad de DLP-2462 menor frente a la del Solapa.

3. La tendencia incremental del clon DLP-2462 nos permite afirmar que a medida que se cultiva más, mejor se adapta a las condiciones medioambientales del valle Quiroz, y sus rendimientos aumentan; es decir, estamos frente a un caso de adaptación positiva.

4. Los rendimientos promedios de raíces reservantes del clon DLP-2462 son significativamente menores a los del cv. Solapa y las ten-

Tabla 6. Composición nutricional (\%) del forraje seco*

\begin{tabular}{lccc}
\hline Componente & $\begin{array}{c}\text { DLP-2462 } \\
\text { (Lactogénico) }\end{array}$ & $\begin{array}{c}\text { Solapa } \\
\text { (Testigo) }\end{array}$ & $\begin{array}{c}\text { Diferencia } \\
(\%)\end{array}$ \\
\hline Proteína total & $\mathbf{1 0 , 7 5}$ & $\mathbf{7 , 7 7}$ & $\mathbf{3 8 , 5}$ \\
Extracto etéreo & 2,27 & 3,05 & $(25,6)$ \\
Fibra cruda & 18,60 & 21,81 & $(14,7)$ \\
Ceniza & 7,54 & 6,91 & 9,1 \\
ELN (1). & 48,35 & 47,27 & 2,3 \\
Humedad & 12,49 & 13,19 & $(5,3)$ \\
Total (\%) & 100,00 & 100,00 & ---- \\
\hline (*)Análisis LENA 0801/2006-UNA-La Molina/(1)Extracto libre de
\end{tabular}
nitrógeno.

\section{Evaluación nutricional}

\section{Composición nutricional del forraje}

El clon DLP-2462, de demostrada aptitud forrajera, produce forraje y raíces reservantes para alimentación animal principalmente, por lo que es imprescindible el conocimiento de las bondades nutricionales del forraje, preparándose muestras representativas para análisis en laboratorios especializados. Los resultados del análisis de laboratorio de la composición nutricional se muestran en la Tabla 6. (Análisis LENA 0801/2006 - Universidad Nacional Agraria La Molina).

1. El contenido de proteína es de $10,75 \%$ y 7,77 \% para DLP-2462 y Solapa respectivamente.

2. Este mayor contenido de $38,5 \%$ de proteína total constituye la principal ventaja competitiva nutricional frente al cv. Solapa, lo que muestra la mejor bondad cualitativa del clon DLP-2462 como fuente proteica para alimentación especialmente del ganado lechero caprino.

3. El mayor contenido de ceniza $(9,1 \%)$ de DLP2462 constituye otra ventaja competitiva frente al cv. Solapa, como fuente de minerales para la alimentación del ganado.

4. El extracto libre de nitrógeno (ELN), base de los carbohidratos, es de $48,35 \%$ y $47,27 \%$ 
para DLP-2462 y Solapa, lo que muestra las buenas cualidades como fuentes energéticas.

\section{Pruebas de palatabilidad y digestibilidad del forraje.}

La palatabilidad de un forraje es el consumo preferencial que tiene el animal por uno u otro alimento.

La digestibilidad de un forraje comprende las modificaciones que sufre éste en el tracto digestivo y que lo prepara para su absorción y utilización por el organismo del animal (en este caso de caprinos).

Para determinar ambos parámetros, se hicieron pruebas de alimentación en un hato de caprinos criollos adultos de ambos sexos del valle Quiroz, lográndose los siguientes resultados:

- La palatabilidad del clon DLP-2462 resulta significativamente superior en comparación con el cv. Solapa.

- La digestibilidad diferencial del clon DLP-2462 y el cultivar Solapa es:

- La digestibilidad aparente de proteína es de $80,14 \%$ para DLP-2462 y $74,3 \%$ para Solapa.

- La digestibilidad de la fibra es 38,6 y 40,6 $\%$, para DLP-2462 y Solapa respectivamente; estos resultados se pueden calificar como niveles bajos.

- La digestibilidad del extracto etéreo y de ELN presentaron porcentajes relativamente altos, no habiendo diferencias marca-
DLP-2462 y Solapa respectivamente, considerados como buenos niveles de digestibilidad.

\section{Composición nutricional (\%) de raíces reser- vantes}

El Análisis de la Composición Nutricional de raíces reservantes de DLP-2462 no se realizo debido a su insignificante nivel de producción.

\section{Materia seca de follaje y raíces reservantes}

El potencial del forraje de camote forrajero está dado por su contenido de materia seca, por cuanto el valor nutricional del forraje se expresa en términos de materia seca.

Evaluaciones para determinar la materia seca del follaje alcanzan a 18,0 \% y 20,6 \% para el clon DLP-2462 y Solapa respectivamente. Es decir la cuarta parte (1/4) de toda la producción de biomasa vegetal es materia seca alimenticia aprovechable para alimentación.

La materia seca de raíces reservantes (camote comestible) es de 27,5 y $21,8 \%$ para el clon DLP-2462 y Solapa respectivamente. Esta es otra ventaja comparativa de DLP-2462 frente a Solapa en materia seca alimenticia.

\section{Evaluación - validación económica}

La evaluación económica realizada tiene como objetivo validar las bondades económicas (rentabilidad) del clon DLP-2462 comparado con

Tabla 8a. DLP2462: Resumen de costos de producción: PC-105 (s/.x ha)

\begin{tabular}{lccccc}
\hline Rubros & Unidad & Cantidad & P.U. & Parcial & $\%$ \\
\hline 1) Alquiler terreno & ha. & 1 & 400 & 400 & 18 \\
2) Mano de obra & jornal & 40 & 12 & 480 & 21 \\
3) Tracción animal & día/cab & 19 & 27 & 513 & 23 \\
4) Semilla + insumos + agua & global & 1 & 528 & 528 & 23 \\
5) Sacos + adherentes & global & 1 & 3 & 3 & 00 \\
6) Imprevistos & global & 1 & 27 & 27 & 01 \\
7) Intereses + A. Técnica & global & 1 & 299 & 299 & 13 \\
Total x Rubros &.-- &.-- &.-- & 2250 & 100 \\
\hline
\end{tabular}

das entre los dos clones.

- La digestibilidad de materia seca es de 70,8 y $70,9 \%$, para DLP-2462 y Solapa respectivamente.

- Los resultados de Nutrientes Digestibles Totales (NDT) son de 65,1 y $64,5 \%$, para el cv. Solapa que cultivan los agricultores tradicionalmente. Para ello se registró paso a paso todos los ítems componentes de los costos directos y costos indirectos de las Parcelas de Comprobación PC-105 (El Torno) y PC-405 (San Joaquín). Para la evaluación económica del nuevo cultivar 
y el testigo se utilizó la metodología establecida en el Manual de Validación Económica de la oficina de Agroeconomía del INIA (Mencionado por J. P. Molina 2001), Con dicha información se hace el Análisis Económico y Análisis de Rentabilidad comparativa del clon DLP-2462 y del cv. Solapa, lo que permite conocer las bondades económicas.

\section{Estructura de costos de producción}

Esta estructura incorpora los costos directos e indirectos de todo el proceso productivo por hectárea y se obtuvieron de las parcelas de comprobación PC-105 y PC-405, tablas 8a,8b, 9a y 9b. Todo está detallado en el Anexo 4.

\section{Costos e ingresos}

El costo de producción promedio para las dos localidades es de S/. 2322 y de S/. 2 499/ha para el Clon DLP-2462 y cv. Solapa respectivamente; se debe indicar que se incluye costos de asistencia técnica que brinda el proyecto actualmente y que en futuro los productores tendrán que asumirlos directamente. Detalle en la tabla 8.

\section{Ingresos netos}

El ingreso neto promedio del clon DLP2462 es S/. 1869 y del cV. Solapa es S/. 2 876, y se observa una diferencia de ingresos de $\mathrm{S} / .1$ 007. Tabla 9.

Tabla 8b. Solapa: Resumen de costos de producción: PC-105 (s/.x ha)

\begin{tabular}{lccccc}
\hline Rubros & Unidad & Cantidad & P.U. & Parcial & $\%$ \\
\hline 1) Alquiler terreno & ha. & 1 & 400 & 400 & 16 \\
2) Mano de obra & jornal & 52 & 12 & 624 & 24 \\
3) Tracción animal & día/cab & 24 & 27 & 648 & 25 \\
4) Semilla + insumos + agua & global & 1 & 528 & 528 & 21 \\
5) Sacos + adherentes & global & 1 & 45 & 45 & 02 \\
6) Imprevistos & global & 1 & 26 & 26 & 01 \\
7) Intereses + A. Técnica & global & 1 & 299 & 299 & 12 \\
Total x Rubros &.-- &.-- &.-- & 2570 & 100 \\
\hline
\end{tabular}

Tabla 9a. DLP-2462: Resumen de costos de producción: PC-405 (s/.x ha)

\begin{tabular}{lccccc}
\hline Rubros & Unidad & Cantidad & P.U. & Parcial & $\%$ \\
\hline 1) Alquiler terreno & ha. & 1 & 400 & 400 & 17 \\
2) Mano de obra & jornal & 42 & 12 & 504 & 21 \\
3) Tracción animal & día/cab & 16 & 27 & 432 & 18 \\
4) Semilla + insumos + agua & global & 1 & 622 & 622 & 26 \\
5) Sacos + adherentes & global & 1 & 10 & 10 & 00 \\
6) Imprevistos & global & 1 & 26 & 26 & 01 \\
7) Intereses + A. Técnica & global & 1 & 400 & 400 & 17 \\
Total x Rubros &.-- &.-- &.-- & 2394 & 100 \\
\hline
\end{tabular}

Tabla 9b. Solapa: Resumen de costos de producción: PC-405 (s/.x ha)

\begin{tabular}{lccccc}
\hline Rubros & Unidad & Cantidad & P.U. & Parcial & $\%$ \\
\hline 1) Alquiler terreno & ha. & 1 & 400 & 400 & 16 \\
2) Mano de obra & jornal & 38 & 12 & 456 & 19 \\
3) Tracción animal & día/cab & 17 & 27 & 459 & 19 \\
4) Semilla + insumos + agua & global & 1 & 672 & 672 & 28 \\
5) Sacos + adherentes & global & 1 & 05 & 05 & 00 \\
6) Imprevistos & global & 1 & 26 & 26 & 01 \\
7) Intereses + A. Técnica & global & 1 & 410 & 410 & 17 \\
Total x Rubros &.-- &.-- &.-- & 2428 & 100 \\
\hline
\end{tabular}


Tabla 8. Costos e ingresos (S/. x ha).

\begin{tabular}{lcccccc}
\hline \multirow{2}{*}{ Lugares } & \multicolumn{2}{c}{ Rendimientos } & \multicolumn{2}{c}{ Costos } & \multicolumn{2}{c}{ Ingresos } \\
& DLP-2462 & Solapa & DLP-2462 & Solapa & DLP-2462 & Solapa \\
\hline El Torno & 39000 & 44700 & 2250 & 2570 & 3539 & 4815 \\
San Joaquín & 53700 & 62400 & 2394 & 2428 & 4843 & 5935 \\
Promedio & 46350 & 53550 & 2322 & 2499 & 4191 & 5375 \\
Desv. Standard & 10395 & 12516 & 101,8 & 100,4 & 922,1 & 792,0 \\
C.V. \% & 22,4 & 23,4 & 4,4 & 4,0 & 22,0 & 14,7 \\
\hline
\end{tabular}

Tabla 9. Ingresos netos (s/. x ha)

\begin{tabular}{lcccc}
\hline \multicolumn{1}{c}{ Lugares } & \multicolumn{2}{c}{ Ingreso Neto } & \multicolumn{2}{c}{ Diferencia de: } \\
& DLP-2462 & Solapa & Ingresos & Costos \\
\hline El Torno & 1289 & 2245 & -956 & -320 \\
San Joaquín & 2449 & 3507 & -1058 & -34 \\
Promedio & 1869 & 2876 & 1007 & -177 \\
\hline
\end{tabular}

El precio promedio de camote en chacra entre ago 2005-jul 2006 fue de S/. 0,20-0,25 x kg, en el valle Quiroz.

\section{Rentabilidad directa y marginal}

La rentabilidad directa promedio del clon DLP-2462 y la del cultivar Solapa son de 79,8 y $115,9 \%$, siendo prácticamente iguales y con una rentabilidad marginal positiva de $1706 \%$. Tabla 10.
Análisis de sensibilidad de rendimientos y costos.

\section{a. Sensibilidad del rendimiento}

Este análisis determina el rendimiento mínimo que el agricultor debe obtener para lograr ingresos iguales a sus costos y conocer cuál es la probabilidad de esta ocurrencia. Detalle en la tabla 12.

Tabla 10. Rentabilidad directa y marginal (S/. x ha).

\begin{tabular}{lccc}
\hline \multirow{2}{*}{ Lugares } & \multicolumn{2}{c}{ Rentabilidad Directa \% } & \multirow{2}{*}{ Rentabilidad Marginal \% } \\
& DLP-2462 & Solapa & 299 \\
\hline El Torno & 57,3 & 87,4 & 3112 \\
San Joaquín & 102,3 & 144,4 & 1706 \\
Promedio & 79,8 & 115,9 & \\
\hline
\end{tabular}

\section{Análisis de riesgo}

En el Cuadro 11, se observa para el clon DLP-2462 un c.v. de 34,$0 ; 4,7$ y $20,7 \%$, mientras que el cv. Para Solapa 23,$4 ; 4,0$ y $14,7 \%$, para los rendimientos, costos e ingresos respectivamente, siendo ligeramente mayor el riesgo del clon DLP2462 debido a su condición de clon introducido, lo que se superará progresivamente a medida que se continúe con su explotación en el valle y se logre una mejor adaptación al medio ambiente.
Los resultados indican que el agricultor debe obtener un rendimiento mínimo de 16,0 t/ha sembrando DLP-2462. Con dicho rendimiento la probabilidad de pérdida es de $0,0 \%$ y con Solapa debe obtener $24,9 \mathrm{t} / \mathrm{ha}$, y con dicho rendimiento la probabilidad de pérdida es de $0,0 \%$. Es decir, la probabilidad de que se produzcan pérdidas logrando los rendimientos indicados con DLP-2462 o Solapa es nula. 
Tabla 11. Análisis de riesgo (s/. ha)

\begin{tabular}{lcccccc}
\hline \multicolumn{1}{c}{ Ítems } & DLP-2462 & & \multicolumn{3}{c}{ Solapa } \\
& Rendición & Costos & Ingresos & Rendición & Costos & Ingresos \\
\hline Promedio & 46350 & 2322,0 & 4191,0 & 53,550 & 2499,0 & $5,375,0$ \\
Desv. Stand. & 10395 & 101,8 & 922,1 & 12515,8 & 100,4 & 792,0 \\
C. V. (\%) & 34,0 & 4,4 & 20,7 & 23,4 & 4,0 & 14,7 \\
\hline
\end{tabular}

Tabla 12. Sensibilidad del rendimiento

\begin{tabular}{llllll}
\hline Clones & $\begin{array}{l}\text { Rendición } \\
\text { mínima }(\mathrm{t} / \mathrm{ha})\end{array}$ & $\begin{array}{l}\text { Sensibilidad } \\
\text { ingresos }\end{array}$ & $\begin{array}{l}\text { Probabili- } \\
\text { dad Ocur. }\end{array}$ & $\begin{array}{l}\text { Sensibili- } \\
\text { dad costos }\end{array}$ & $\begin{array}{l}\text { Probabilidad } \\
\text { Ocur. }\end{array}$ \\
\hline DLP-2462 & 16014 & $-2,23$ & $0,0 \%$ & 0,80 & $0,0 \%$ \\
Solapa & 24990 & $-2,28$ & $0,0 \%$ & 1,15 & $0,0 \%$ \\
\hline
\end{tabular}

\section{b. Sensibilidad de Costos}

En este análisis se determina en qué porcentaje se elevarían los costos, manteniendo los ingresos constantes para llegar a producir pérdidas al agricultor.

La probabilidad de que el costo de producción se eleve en $8 \%$ sembrando el clon DLP-2462 es 0,0\%; y la probabilidad de que el costo de producción se eleve en $15 \%$ sembrando el cultivar Solapa es $0,0 \%$.

La evaluación de rentabilidad económica del cultivo permite mostrar que con la siembra del clon DLP-2462 se obtienen ingresos iguales que con Solapa, por la continuidad de la producción y el mayor precio que recibe en el mercado durante el año frente al cultivar Solapa.
El clon DLP-2462 se puede sembrar exitosamente durante todo el año. Esta es una ventaja comparativa del clon DLP2462 frente al cultivar Solapa que solo produce buenos rendimientos en épocas clásicas de siembras (abril, mayo).

\section{Ratio: Beneficio / Costo}

Este exigente parámetro de evaluación económica muestra que el clon DLP2462 alcanza una relación $\mathrm{R}: \mathrm{B} / \mathrm{C}$ de 1,80 en promedio, lo que significa que por cada sol invertido en el cultivo de DLP-2462 se puede lograr ingresos de S/. 0,80 más, lo que lo hace atractivo al productor y a los posibles inversionistas en el cultivo.

Los ingresos que genera Solapa son mayores, siendo también otra opción económica exitosa.

Tabla 13. Ratio: beneficio / costo.

\begin{tabular}{lcccc}
\hline Experimento - Localidad & Cultivar & Costos & Ingresos & R: B/C \\
\hline PC-105 -E1 Torno & DLP-2462 & 2250,0 & 3539,0 & 1,57 \\
PC-405 - San Joaquín & DLP-2462 & 2394,0 & 4843,0 & 2,02 \\
Promedio & -- & 2322,0 & 4191,0 & 1,80 \\
PC-105 -El Torno & Solapa & 2570,0 & 4815,0 & 1,87 \\
PC-405 - San Joaquín & Solapa & 2428,0 & 5935,0 & 2,44 \\
Promedio & --- & 2499,0 & 5375,0 & 2,15 \\
\hline
\end{tabular}




\section{CONCLUSIONES}

Los resultados de los Experimentos Intermedios, Avanzados y Parcelas de Comprobación realizados entre 2001-2003, 2004 y 2005 , en cinco localidades del valle QuirozSuyo, Piura, y en distintas épocas de siembracosecha durante el año, permiten concluir lo siguiente:

\section{Evaluaciones agronómicas}

El clon DLP-2462 posee ventajas agronómicas comparativas similares al cv. Solapa en:

1. Rendimientos promedio estables de 40,5 $\mathrm{t} / \mathrm{ha}$ de forraje y 3,0 t/ha de raíces reservantes, en comparación con el cultivar Solapa que obtuvo 43,0 y 5,8 t/ha de forraje y raíces reservantes en 150 días de cultivo.

2. Experimentos en 4 épocas: verano-otoño, otoño-invierno, invierno-primavera $\mathrm{y}$ primavera-verano; durante el año en siembras-cosechas sucesivas, DLP-2462 produjo rendimientos de 40,5 t/ha de forraje y 3,0 t/ha de raíces reservantes. Mostrando excelente adaptación a las condiciones ecológicas y agroeconómicas del valle Quiroz y aledaños.

3. El contenido de materia seca del forraje con $18,0 \%$ y de $27,5 \%$ en raíces reservantes adiciona a las bondades de calidad nutritiva del follaje y raíces.

4. El clon DLP-2462 es lentamente adoptado por los agricultores, quienes están sembrando pequeñas áreas, debido a la calidad nutricional del forraje. (Anexo 2).

5. El proceso de investigación ha permitido generar una moderna Tecnología de Producción de Camote Forrajero de fácil aplicación por los productores de la región norte de Perú. (Anexo 3).

\section{Evaluaciones nutricionales}

1. Las cualidades nutricionales del fol- laje del clon DLP-2462 son apreciables, porque posee $10.75 \%$ de proteína en seco (heno), constituyéndose en una excelente fuente proteica de bajo costo para la alimentación de todo tipo de ganado por ser un forraje versátil.

2. El clon DLP-2462 contiene $38,5 \%$ más de proteína que Solapa, lo que constituye la mejor ventaja competitiva del clon como fuente proteica para alimentación animal.

3. Tiene superior palatabilidad y digestibilidad para el ganado caprino.

4. La palatabilidad del clon DLP-2462 es mayor que la del Solapa, y tiene una digestibilidad con NDT de 65,1 y 64,5\% respectivamente, lo que ratifica sus bondades nutricionales y aptitud lactogénica.

\section{Evaluaciones económicas}

1. El clon DLP-2462 alcanza S/.1 869,0 de ingresos netos y S/.2 876,0 el cultivar Solapa.

2. Los rendimientos mínimos que deben alcanzar los agricultores para no tener pérdidas son de 22.33 y $24,99 \mathrm{t} / \mathrm{ha}$ con DLP-2462 y Solapa respectivamente, no existiendo ninguna probabilidad de pérdidas con esos rendimientos obtenidos. (probabilidad nula).

3. El clon DLP-2462 alcanza una relación $\mathrm{R}$ : $\mathrm{B} / \mathrm{C}$ de 1,80 en promedio, lo que significa que por cada sol invertido en el cultivo se puede lograr ingresos adicionales de S/. 0,80 más, lo que lo hace atractivo al productor y a los posibles inversionistas, constituyendo este cultivo una nueva opción de inversión económica. (Anexo 4).

\section{SUGERENCIAS}

Se sugiere que, en relación con el clon DLP-2462 como nuevo cultivar lactogénico, se inicie la masiva producción de núcleos 
de semilla de calidad sanitaria mediante un sistema de producción de semilla garantizada para los agricultores, y paralelamente ingrese a un proceso de refrescamiento sanitario para contar con plantas madres.

\section{RECOMENDACIÓN}

Las superiores ventajas agronómicas, nutricionales y económicas expuestas, permiten recomendar:

La liberación del clon DLP-2462 como nuevo cultivar lactogénico, de aptitud forrajera, y su promoción y difusión masiva como cultivo alternativo viable para agricultura en bosques secos del valle Quiroz y de la región norte del Perú.

\section{REFERENCIAS BIBLIOGRÁFICAS}

1. BEAUFORT-MURPHY, H. 1994. "Desarrollo de una variedad de camote (Ipomoea batatas) con amplia adaptación como nuevo cultivo forrajero perenne, ambientalmente sostenible". In: Resúmenes VIII Congreso Internacional de Sistemas Agropecuarios y su proyección al tercer milenio. Valdivia, Chile. \#1994, p.17.\#.22.

2. BARRIOS, J. R. y COLMENARES R. 1989. Potencialidad de la batata (Ipomoea batatas (L) Lam.) como forraje verde. Alcance 38. Revista de la Facultad de Agronomía. U. C. V. Maracay-Venezuela.

3. CIP 2000. Procedures for the evaluation of Internad-tested sweetpotato clones. Lima, Perú.
4. CIP-CARE, 2002. Guía para facilitar el desarrollo de escuelas de campo de agricultores. Lima, Perú.

5. GÓMEZ, K.A. \& Gómez, A. A. 1983. Statistical procedures for agricultural research. 2nd. Edition, New York.

6. INIA. 2000. Normas para el lanzamiento de nuevo cultivar. Resolución Jefatural $\mathrm{N}^{\circ}$ 020-2000.INIA-AG.

7. INIA. 2001. Informe Técnico y Validación Económica de la nueva Variedad de Camote HUAMBACHERO-INIA. E.E. D'onoso. Intern.

8. MOLINA, J. P. 2004. Manual de Validación Económica de la oficina de Agroeconomía del INIA-Lima.

9. MINAG-PIURA. 2000. Plan de desarrollo ganadero para el departamento de Piura. 2000-2015. Piura-Perú.

10. QUISPE, C. A. 2002. Evaluación de clones forrajeros y de doble propósito por adaptación y rendimiento en trópicos secos de Piura. CIDA PERÚ, Piura. Documento de Trabajo.

11. ROCA, T. J., De Mendiburu, F. y LeónVelarde, C.U. 2002. Selección de accesiones de camote (Ipomoea batatas L.) con características de doble propósito. Análisis de evaluaciones agronómicas (1994-2002). Documento de Trabajo. Octubre 2001, La Molina.

12. SÁNCHEZ, V. H. 1996. Valor nutricional del ensilaje de raíces no comerciales $y$ follaje de camote. Tesis Magíster Scientiae, UNA La Molina, Lima-Perú.

13. Seminario, N. H. 2004. Caracterización de la Tecnología Local de Producción de Camote en el Valle Quiroz - Piura CIDA - PERÚ. 


\section{Anexos}

ANEXO 1. DATA DE COLECCIÓN, CARACTERIZACIÓN DEL CLON DLP-2462. ANEXO 2. COSTOS DE PRODUCCIÓN DE PARCELAS DE COMPROBACIÓN.

\section{Anexo 1. Data de colección, caracterización del clon DLP-2462.}

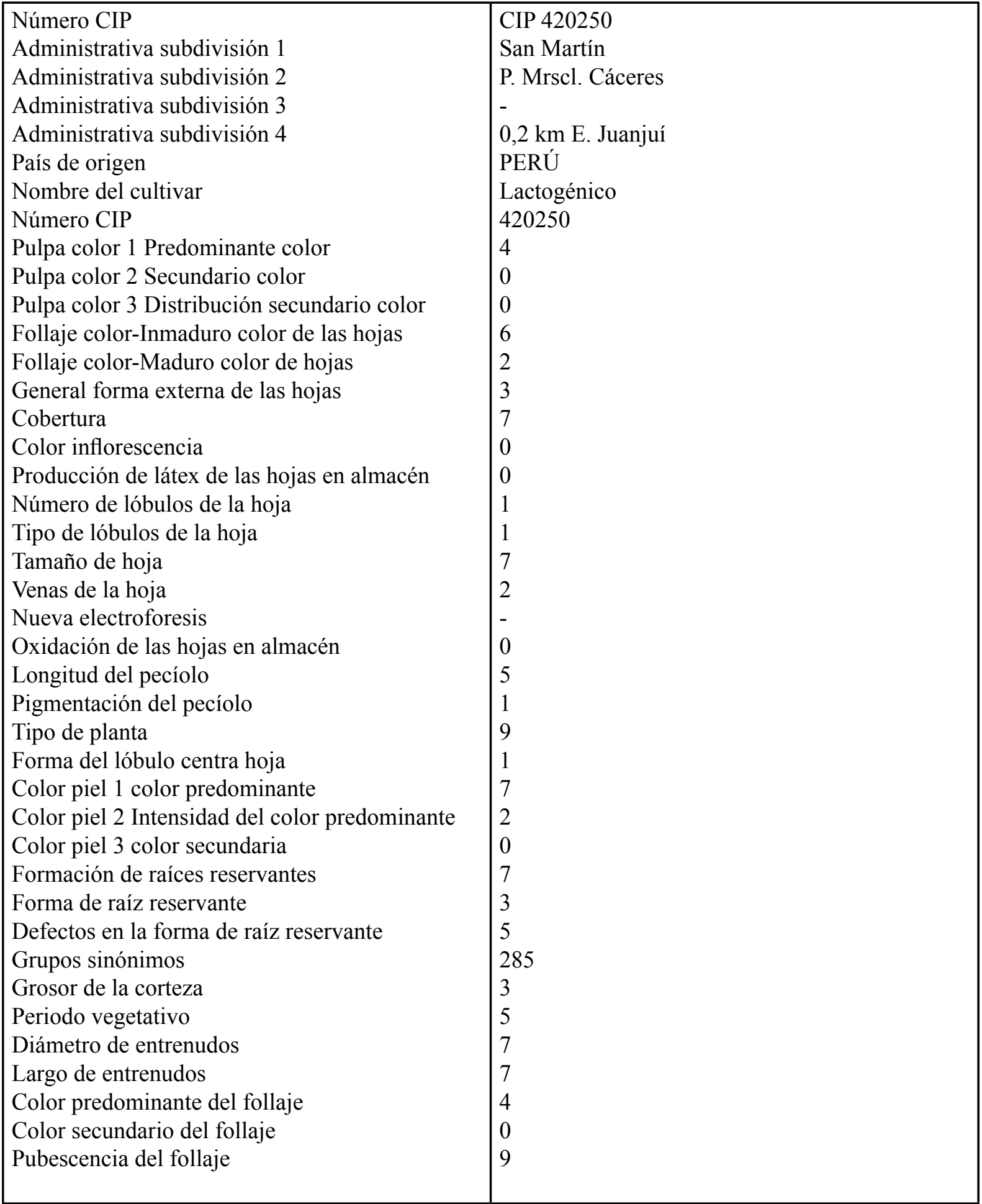


Anexo 2. costos de producción de parcelas de comprobación PC-105 y PC-405

CIDA Perú

ANEXO 2.A

Proyecto camote forrajero

\begin{tabular}{llll} 
& \multicolumn{3}{l}{ Costos de producción: PC-105 (s/. x ha). } \\
& DEPARTAMENTO & Piura \\
VALLE/SECTOR & $:$ & Quiroz / El Torno & \\
CAMPAÑA & $:$ & 150 días $(27 / 3-27 / 8 / 05)$ \\
PERIODO & $:$ & Otoño Invierno \\
TECNOLOGÍA & $:$ & Mejorada \\
DENSIDAD & $:$ & 33350 plantas \\
VARIEDAD & $:$ & DLP-2462 LACTOGÉNICO
\end{tabular}

Resumen de costos

\begin{tabular}{|c|c|c|c|c|c|}
\hline Rubros & Unid. & Cant. & P.U. & Parcial & $\%$ \\
\hline 1) Alquiler terreno & ha. & 1 & 400 & 400 & 18 \\
\hline 2) Mano de obra & jornal & 40 & 12 & 480 & 21 \\
\hline 3) Tracción animal & día/cab & 19 & 27 & 513 & 23 \\
\hline 4) Semilla + insumos + agua global & global & 1 & 528 & 528 & 23 \\
\hline 5) Sacos + adherentes global & global & 1 & 3 & 3 & 0 \\
\hline 6) Imprevistos global & global & 1 & 27 & 27 & 1 \\
\hline 7) Intereses $+A$. Técnica & global & 1 & 299 & 299 & 13 \\
\hline Total x Rubros &.-- &.-- &.-- & 2250 & 100 \\
\hline
\end{tabular}

C. Directos $=1,2,3,4,5$ y 6; Indirectos $=7$.

Análisis económico

\begin{tabular}{|c|c|c|c|}
\hline ITEMS & UNID. & & MONTOS \\
\hline COSTOS DIRECTOS & $(\mathrm{S} /)$. & : & 1951 \\
\hline COSTOS INDIRECTOS & $(\mathrm{S} /)$. & : & 299 \\
\hline COSTO TOTAL & $(\mathrm{S} /)$. & : & 2250 \\
\hline COSTO BRUTO FORRAJE & $(\mathrm{S} /)$. & : & 2093 \\
\hline COSTO BRUTO CAMOTE & $(\mathrm{S} /)$. & : & 158 \\
\hline RENDIMIENTO FORRAJE & (kg./ha) & : & 36100 \\
\hline RENDIMIENTO CAMOTE & (kg./ha) & : & 2900 \\
\hline RENDIMIENTO TOTAL & (kg./ha) & : & 39000 \\
\hline COSTO UNITARIO-FORRAJE & $(\mathrm{S} / . \mathrm{x} \mathrm{kg})$ & : & 0,058 \\
\hline COSTO UNITARIO-CAMOTE & $(\mathrm{S} / . \mathrm{x} \mathrm{kg})$ & : & 0,054 \\
\hline PRECIO VENTA FORRAJE & $(\mathrm{S} / . \mathrm{x} \mathrm{kg})$ & : & 0,09 \\
\hline PRECIO VENTA CAMOTE & $(\mathrm{S} / . \mathrm{x} \mathrm{kg})$ & : & 0,10 \\
\hline INGRESO x FORRAJE & $(\mathrm{S} /)$. & : & 3249 \\
\hline INGRESO x CAMOTE & $(\mathrm{S} /)$. & : & 290 \\
\hline INGRESO TOTAL $(\mathrm{F}+\mathrm{C})$ & & & 3539 \\
\hline UTILIDAD NETA C/FORRAJE & $(\mathrm{S} /)$. & : & 1156 \\
\hline UTILIDAD NETA C/CAMOTE & $(\mathrm{S} /)$. & : & 132 \\
\hline UTILIDAD NETA TOTAL & $(\mathrm{S} /)$. & : & 1289 \\
\hline INGRESO NETO DIARIO & (S/. x día) & : & 8,59 \\
\hline R: B/C -FORRAJE + CAMOTE & Índice & : & 1,57 \\
\hline
\end{tabular}


CIDA PERU

ANEXO 2.B

Proyecto camote forrajero

Costos de producción: PC-405 (s/. x ha).

$\begin{array}{llll}\text { DEPARTAMENTO } & : & \text { Piura } \\ \text { VALLE / SECTOR } & : & \text { Quiroz / San Joaquín } \\ \text { CAMPAÑA } & : & & \text { 190 días 15/11/05 - 25/5/06 } \\ \text { PERIODO } & : & \text { Primavera } & \text { Verano } \\ \text { TECNOLOGIA } & : & \text { Mejorada } \\ \text { DENSIDAD } & : & 40,000 \text { plantas } \\ \text { VARIEDAD } & : & \text { DLP-2462 - LACTOGENICO }\end{array}$

Resumen de costos

\begin{tabular}{l|c|c|c|c|c|}
\hline \multicolumn{1}{|c|}{ Rubros } & Unid. & Cant. & P.U. & Parcial & $\%$ \\
\hline 1) Alquiler terreno & ha. & 1 & 400 & 400 & 17 \\
2) Mano de obra & jornal & 42 & 12 & 504 & 21 \\
3) Tracción animal & día/cab & 16 & 27 & 432 & 18 \\
4) Semilla + insumos + agua & global & 1 & 622 & 622 & 26 \\
5) Sacos + adherentes & global & 1 & 10 & 10 & 00 \\
6) Imprevistos & global & 1 & 26 & 26 & 01 \\
7) Intereses + A. Técnica & global & 1 & 400 & 400 & 17 \\
$\quad$ Total x Rubros &.-- &.-- &.-- & 2394 & 100 \\
\hline
\end{tabular}

C. Directos $=1,2,3,4,5$ y 6; Indirectos $=7$.

\section{Análisis económico}

\begin{tabular}{|c|c|c|}
\hline ITEMS & UNID. & MONTOS \\
\hline COSTOS DIRECTOS & $(\mathrm{S} /)$. & 1994 \\
\hline COSTOS INDIRECTOS & $(\mathrm{S} /)$. & 400 \\
\hline COSTO TOTAL & $(\mathrm{S} /)$. & 2394 \\
\hline COSTO BRUTO FORRAJE & $(\mathrm{S} /)$. & 2346 \\
\hline COSTO BRUTO CAMOTE & $(\mathrm{S} /)$. & 48 \\
\hline RENDIMIENTO FORRAJE & (kg./ha) & 52700 \\
\hline RENDIMIENTO CAMOTE & (kg./ha) & 1000 \\
\hline RDTO (FORRAJE+CAMOTE) & (kg./ha) & 53700 \\
\hline COSTO UNITARIO-FORRAJE & $(\mathrm{S} / . \mathrm{x} \mathrm{kg})$ & 0,045 \\
\hline COSTO UNITARIO-CAMOTE & $(\mathrm{S} / . \mathrm{x} \mathrm{kg})$ & 0,048 \\
\hline PRECIO VENTA FORRAJE & $(\mathrm{S} / . \mathrm{x} \mathrm{kg})$ & 0,09 \\
\hline PRECIO VENTA CAMOTE & $(\mathrm{S} / \mathrm{x} \mathrm{kg})$ & 0,10 \\
\hline INGRESO $x$ FORRAJE & $(\mathrm{S} /)$. & 4743 \\
\hline INGRESO x CAMOTE & $(\mathrm{S} /)$. & 100 \\
\hline INGRESO TOTAL $(\mathrm{F}+\mathrm{C})$ & & 4843 \\
\hline UTILIDAD CON FORRAJE & $(\mathrm{S} /)$. & 2397 \\
\hline UTILIDAD CON CAMOTE & $(\mathrm{S} /)$. & 52 \\
\hline UTILIDAD NETA TOTAL & $(\mathrm{S} /)$. & 2449 \\
\hline INGRESOS DIARIOS & (S/. x día) & 12,89 \\
\hline R: B/C; FORRAJE+CAMOTE & Índice & 2,02 \\
\hline
\end{tabular}


CIDA PERU

ANEXO 2.C

Proyecto camote forrajero

Costos de producción: PC-105 (s/. x ha).

$\begin{array}{lll}\text { DEPARTAMENTO } & : & \text { Piura } \\ \text { VALLE/SECTOR } & : & \text { Quiroz / El Torno } \\ \text { CAMPAÑA } & : & 150 \text { días }(27 / 3-27 / 8 / 05) \\ \text { PERIODO } & : & \text { Otoño Invierno } \\ \text { TECNOLOGIA } & : & \text { Mejorada } \\ \text { DENSIDAD } & : & 33,350 \text { plantas } \\ \text { VARIEDAD } & : & \text { SOLAPA }\end{array}$

Resumen de costos

\begin{tabular}{l|c|c|c|c|c|}
\hline \multicolumn{1}{|c|}{ Rubros } & Unid. & Cant. & P.U. & Parcial & $\%$ \\
\hline 1) Alquiler terreno & ha. & 1 & 400 & 400 & 16 \\
2) Mano de obra & jornal & 52 & 12 & 624 & 24 \\
3) Tracción animal & día/cab & 24 & 27 & 648 & 25 \\
4) Semilla + insumos + agua & global & 1 & 528 & 528 & 21 \\
5) Sacos + adherentes & global & 1 & 45 & 45 & 02 \\
6) Imprevistos & global & 1 & 26 & 26 & 01 \\
7) Intereses + A. Técnica & global & 1 & 299 & 299 & 12 \\
\multicolumn{1}{c}{ Total x Rubros } &.-- &.-- &.-- & 2570 & 100 \\
\hline
\end{tabular}

C. Directos $=1,2,3,4,5$ y 6; Indirectos $=7$.

Análisis económico

\begin{tabular}{|c|c|c|c|}
\hline ITEMS & UNID. & & MONTOS \\
\hline COSTOS DIRECTOS & $(\mathrm{S} /)$. & : & 2271 \\
\hline COSTOS INDIRECTOS & $(\mathrm{S} /)$. & : & 299 \\
\hline COSTO TOTAL & $(\mathrm{S} /)$. & : & 2570 \\
\hline COSTO BRUTO FORRAJE & $(\mathrm{S} /)$. & : & 2159 \\
\hline COSTO BRUTO CAMOTE & $(\mathrm{S} /)$. & : & 411 \\
\hline RENDIMIENTO FORRAJE & (Kg./ha) & : & 37500 \\
\hline RENDIMIENTO CAMOTE & (Kg./ha) & : & 7200 \\
\hline RENDIMIENTO TOTAL & (Kg./ha) & : & 44700 \\
\hline COSTO UNITARIO-FORRAJE & $(\mathrm{S} / . \mathrm{x} \mathrm{Kg})$ & : & 0,058 \\
\hline COSTO UNITARIO-CAMOTE & $(\mathrm{S} / . \mathrm{x} \mathrm{Kg})$ & : & 0,057 \\
\hline PRECIO VENTA FORRAJE & $(\mathrm{S} / . x \mathrm{Kg})$ & : & 0,09 \\
\hline PRECIO VENTA CAMOTE & $(\mathrm{S} / . \mathrm{x} \mathrm{Kg})$ & : & 0,20 \\
\hline INGRESO x FORRAJE & $(\mathrm{S} /)$. & : & 3375 \\
\hline INGRESO x CAMOTE & $(\mathrm{S} /)$. & : & 1440 \\
\hline INGRESO TOTAL $(\mathrm{F}+\mathrm{C})$ & & & 4815 \\
\hline UTILIDAD NETA C/FORRAJE & $(\mathrm{S} /)$. & : & 1216 \\
\hline UTILIDAD NETA C/CAMOTE & $(\mathrm{S} /)$. & : & 1029 \\
\hline UTILIDAD NETA TOTAL & $(\mathrm{S} /)$. & : & 2245 \\
\hline INGRESO NETO DIARIO & (S/. x día) & : & 14,97 \\
\hline R: B/C -FORRAJE + CAMOTE & Índice & : & 1,87 \\
\hline
\end{tabular}


CIDA PERU

ANEXO 2.D

Proyecto camote forrajero

Costos de producción: PC-405 (s/. x ha).

$\begin{array}{llll}\text { DEPARTAMENTO } & : & & \text { Piura } \\ \text { VALLE / SECTOR } & : & & \text { Quiroz / San Joaquín } \\ \text { CAMPAÑA } & : & & 190 \text { días } 15 / 11 / 05-25 / 5 / 06 \\ \text { PERIODO } & : & \text { Primavera } & \text { Verano } \\ \text { TECNOLOGIA } & : & \text { Mejorada } & \\ \text { DENSIDAD } & : & 40,000 \text { plantas } & \\ \text { VARIEDAD } & : & \text { SOLAPA }\end{array}$

Resumen de costos

\begin{tabular}{l|c|c|c|c|c|}
\hline \multicolumn{1}{|c|}{ Rubros } & Unid. & Cant. & P.U. & Parcial & $\%$ \\
\hline 1) Alquiler terreno & ha. & 1 & 400 & 400 & 16 \\
2) Mano de obra & jornal & 38 & 12 & 456 & 19 \\
3) Tracción animal & día/cab & 17 & 27 & 459 & 19 \\
4) Semilla + insumos + agua & global & 1 & 672 & 672 & 28 \\
5) Sacos + adherentes & global & 1 & 5 & 5 & 00 \\
6) Imprevistos & global & 1 & 26 & 26 & 01 \\
7) Intereses + A. Técnica & global & 1 & 410 & 410 & 17 \\
\multicolumn{1}{c}{ Total x Rubros } &.-- &.-- &.-- & 2428 & 100 \\
\hline
\end{tabular}

Análisis económico

\begin{tabular}{|c|c|c|c|}
\hline ITEMS & UNID. & & MONTOS \\
\hline COSTOS DIRECTOS & $(\mathrm{S} /)$. & $:$ & 2018 \\
\hline COSTOS INDIRECTOS & $(\mathrm{S} /)$. & : & 410 \\
\hline COSTO TOTAL & $(\mathrm{S} /)$. & : & 2428 \\
\hline COSTO BRUTO FORRAJE & $(\mathrm{S} /)$. & : & 2307 \\
\hline COSTO BRUTO CAMOTE & $(\mathrm{S} /)$. & $:$ & 121 \\
\hline RENDIMIENTO FORRAJE & (Kg./ha) & $:$ & 59500 \\
\hline RENDIMIENTO CAMOTE & (Kg./ha) & : & 2900 \\
\hline RDTO (FORRAJE+CAMOTE) & (Kg./ha) & : & 62400 \\
\hline COSTO UNITARIO-FORRAJE & $(\mathrm{S} / . \mathrm{x} \mathrm{Kg})$ & $:$ & 0,039 \\
\hline COSTO UNITARIO-CAMOTE & $(\mathrm{S} / . \mathrm{x} \mathrm{Kg})$ & : & 0,042 \\
\hline PRECIO VENTA FORRAJE & $(\mathrm{S} / . \mathrm{x} \mathrm{Kg})$ & : & 0,09 \\
\hline PRECIO VENTA CAMOTE & $(\mathrm{S} / . \mathrm{x} \mathrm{Kg})$ & : & 0,20 \\
\hline INGRESO $\times$ FORRAJE & $(\mathrm{S} /)$. & $:$ & 5355 \\
\hline INGRESO x CAMOTE & $(\mathrm{S} /)$. & : & 580 \\
\hline INGRESO TOTAL $(\mathrm{F}+\mathrm{C})$ & & & 5935 \\
\hline UTILIDAD CON FORRAJE & $(\mathrm{S} /)$. & : & 3048 \\
\hline UTILIDAD CON CAMOTE & $(\mathrm{S} /)$. & $:$ & 459 \\
\hline UTILIDAD NETA TOTAL & $(\mathrm{S} /)$. & $:$ & 3507 \\
\hline INGRESOS DIARIOS & (S/. x día) & : & 18,46 \\
\hline R: B/C; FORRAJE+CAMOTE & Índice & : & 2,44 \\
\hline
\end{tabular}



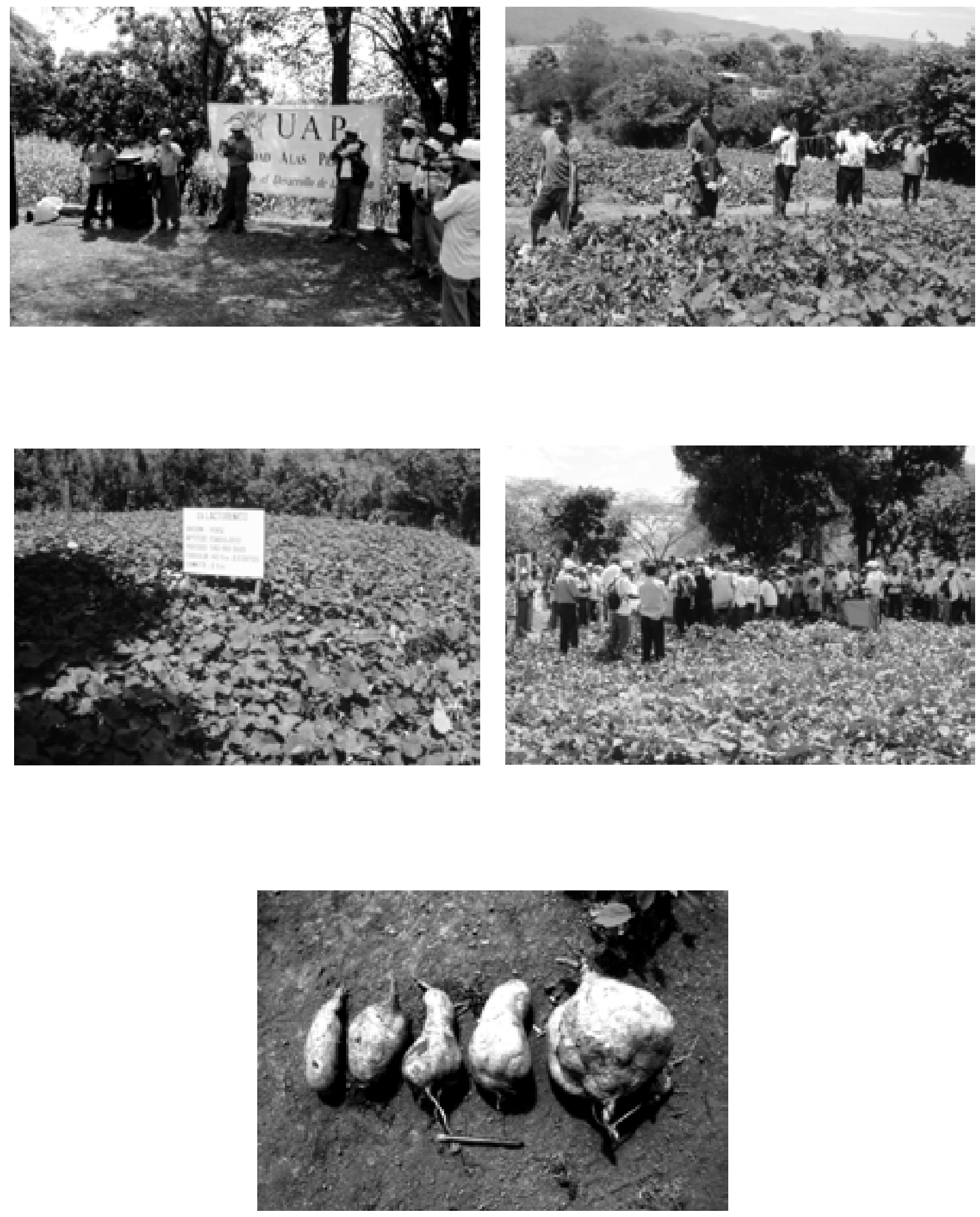